\title{
TMD factorization for dijet and heavy-meson pair in DIS
}

\author{
Rafael F. del Castillo, ${ }^{a}$ Miguel G. Echevarria, ${ }^{b}$ Yiannis Makris $^{c}$ and Ignazio Scimemi ${ }^{a}$ \\ ${ }^{a}$ Departamento de Física Teórica \& IPARCOS, Universidad Complutense de Madrid, \\ E-28040 Madrid, Spain \\ ${ }^{b}$ Departamento de Física y Matemáticas, Universidad de Alcalá, \\ 28805 Alcalá de Henares (Madrid), Spain \\ ${ }^{c}$ INFN Sezione di Pavia, \\ via Bassi 6, I-27100 Pavia, Italy \\ E-mail: raffer06@ucm.es, m.garciae@uah.es, yiannis.makris@pv.infn.it, \\ ignazios@ucm.es
}

ABSTRACT: We study a transverse momentum dependent (TMD) factorization framework for the processes of dijet and heavy-meson pair production in deep-inelastic-scattering in an electron-proton collider, considering the measurement of the transverse momentum imbalance of the two hard probes in the Breit frame. For the factorization theorem we employ soft-collinear and boosted-heavy-quark effective field theories. The factorized crosssection for both processes is sensitive to gluon unpolarized and linearly polarized TMD distributions and requires the introduction of a new soft function. We calculate the new soft function here at one-loop, regulating rapidity divergences with the $\delta$-regulator. In addition, using a factorization consistency relation and a universality argument regarding the heavy-quark jet function, we obtain the anomalous dimension of the new soft function at two and three loops.

KeYWORDS: Jets, QCD Phenomenology

ArXiv EPrint: 2008.07531 


\section{Contents}

1 Introduction 1

2 Dijet imbalance $\quad 4$

2.1 Notation and kinematics 4

$\begin{array}{lll}2.2 & \text { Factorization theorem for dijet production } & 7\end{array}$

2.3 The dijet soft function at NLO 9

$\begin{array}{ll}2.4 \text { The zero-bin subtraction and the universal TMDs } & 12\end{array}$

$\begin{array}{ll}2.5 \text { Consistency check } & 14\end{array}$

3 Heavy-meson pair imbalance $\quad 15$

3.1 Refactorization of heavy-quark fragmentation function 16

$\begin{array}{ll}3.2 & \text { The bHQET matrix element at NLO } \\ & 18\end{array}$

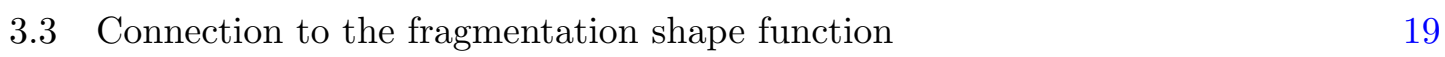

4 Conclusions $\quad 21$

A Elements of factorization $\quad 22$

A.1 Hard function 22

A.2 Jet function 22

A.3 Collinear-soft function 23

$\begin{array}{lll}\text { A.4 Beam function } & 23\end{array}$

A.5 Back-to-back two-direction soft function 24

B Anomalous dimensions $\quad 24$

B.1 The cusp anomalous dimension and $\beta$ function 24

B.2 bHQET heavy-quark jet function $\quad 25$

$\begin{array}{lll}\text { B.3 TMDPDF } & 26\end{array}$

B.4 Hard function 26

$\begin{array}{lll}\text { B.5 Dijet soft function } & 27\end{array}$

$\begin{array}{ll}\text { C Dijet soft function integrals } & 27\end{array}$

\section{Introduction}

It is well known that gluons are an essential constituent of nuclei and that the gluon parton distribution functions (PDFs) can numerically be much bigger than the corresponding quark distributions, especially when the parton energy fraction is small. Gluon transverse momentum dependent distributions (TMDs) are also expected to be similarly enhanced, however they result to be difficult to access due to the lack of clean processes where the 
factorization of the cross-section holds and incoming gluons constitute the dominant effect. An example of such a process is the Higgs production in hadronic colliders [1-5]. However, extractions of gluon TMDs from the Higgs transverse-momentum spectrum is challenging due to the nature of the scalar boson and its large mass (f.i. see also [6] including jet veto considerations and [7] which do not include all TMD effects). Even for the relatively clean process of Higgs spectrum, both the unpolarized and linearly polarized gluon distributions appear in the leading power factorization of the cross-section in the $q_{T} / M_{H}$ expansion, where $q_{T}$ and $M_{H}$ are the boson transverse momentum and mass, respectively. The absence of a color neutral scalar at low energies has driven the attention to quarkonium production both in semi-inclusive deep inelastic scattering (SIDIS) at Electron Ion Collider (EIC) and LHC [3, 8-27]. However, the factorization of these processes is also challenging (see $[22,23]$ ) and a series of QCD effects are present because of the color structure of quarkonia and the complexity of the non-relativistic expansion (commonly used in quarkonium production studies).

In this work we consider two alternative processes which are presently attracting increasing attention: the dijet [28] and heavy-meson pair [29-31] production in an electronhadron collider, as generated by the $\left(\gamma^{*} g\right)$ and/or $\left(\gamma^{*} f\right)$ hard interactions. The processes are

$$
\ell+h \rightarrow \ell^{\prime}+J_{1}+J_{2}+X, \quad \text { and } \quad \ell+h \rightarrow \ell^{\prime}+H+\bar{H}+X,
$$

where $\ell$ and $\ell^{\prime}$ are the initial and final state leptons, $h$ is the colliding hadron, and $J_{i}$ and $H / \bar{H}$ are the jets and heavy mesons, respectively. All undetected particles in (1.1) are represented by $X$.

Dijet production has been the object of several studies at the kinematics of the future electron ion collider (EIC), as it is sensitive to polarized and unpolarized gluon TMDs [32-35]. The produced jets analyzed in the Breit frame have typically a $p_{T} \in$ $[5,40] \mathrm{GeV}$ and are found in the central rapidity region. Recent studies (see for example [35]) suggest that the experimental observation of the dijet imbalance is possible at the future EIC. The kinematic constraints we consider here for the dijet process need to be such that do not create hierarchies among the partonic Mandelstam variables, i.e. we demand that $\hat{s} \sim|\hat{t}| \sim|\hat{u}|$. If such hierarchies exist, they will induce large logarithms in the hard factor of the cross-section and can potentially ruin the convergence of perturbative expansion, unless further resummation/refactorization of the hard factor is performed.

The heavy-meson pair case is instead experimentally more challenging due to the necessity to reconstruct the momenta of the heavy meson from its decay products. In addition, the large energy required to produce a boosted heavy-meson pair makes the process less likely to be observed compared to the dijet production process. On the other hand, recent investigations using monte-carlo generators suggest that for charmed mesons this observable could be possible. From experimental perspective the charm reconstruction have been investigated in refs. [36, 37]. In ref. [38] the charm production rates have been investigated at the LO and NLO QCD for $e p \rightarrow c / \bar{c}+X$. The factorization we construct below requires the transverse momenta of the heavy mesons, $p_{T}^{H / \bar{H}}$, be parametrically larger than their mass, $m_{H}$, i.e. $p_{T}^{H / \bar{H}} \gg m_{H}$, although an alternative factorization can 


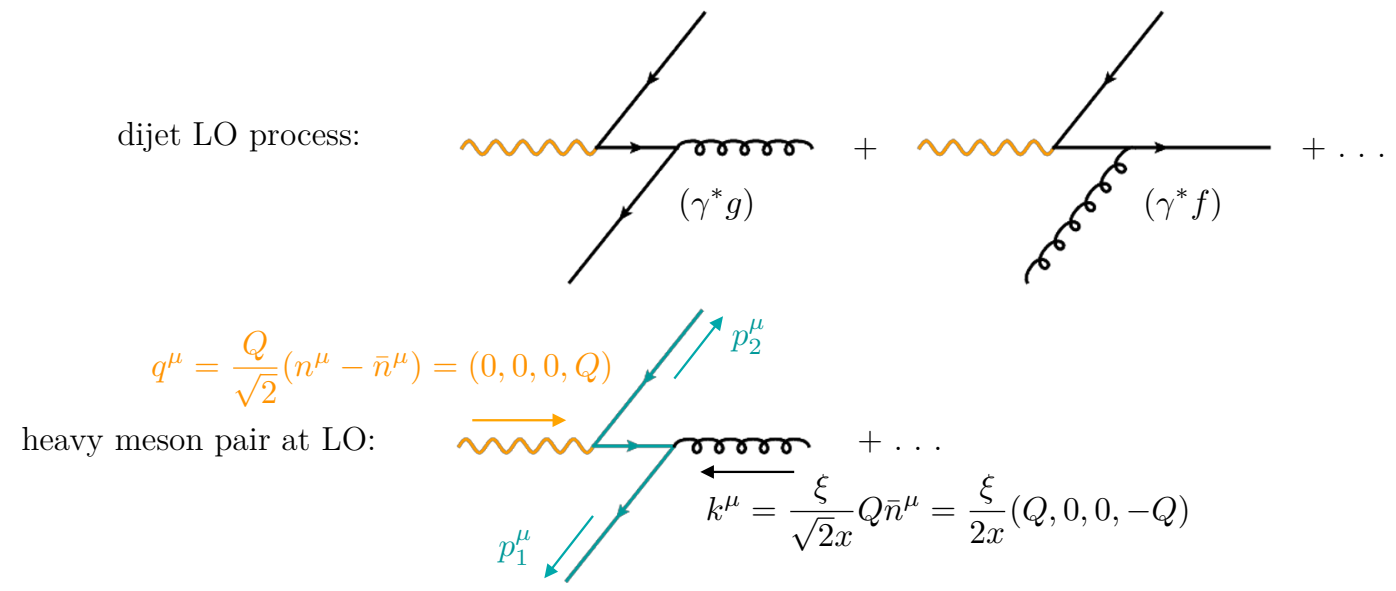

Figure 1. Example LO diagrams for the two processes. The momenta $q^{\mu}$ and $k^{\mu}$ (corresponding to the photon and incoming parton momenta respectively) are expressed in the Breit frame.

be constructed when this condition is violated. The details of such factorization involve a hard function which includes all heavy-quark mass dependence. It also requires a different soft function for which the directions of the quark-antiquark pair are not light-like since the heavy mesons are not boosted to the massless limit. We will not pursue this factorization here, but for a relevant study see ref. [29].

At leading order (LO), and ignoring the intrinsic momentum of partons inside the target hadron, the two hard-scattering processes are schematically shown in figure 1. In the case of jets we have that the initial parton can be either a gluon or a quark, while in the heavy-meson case only the gluon initial state is relevant. ${ }^{1}$ We consider the differential cross-section

$$
\frac{d \sigma}{d x d \eta_{1} d \eta_{2} d p_{T} d \boldsymbol{r}_{T}}
$$

where $x$ is the Bjorken variable, and $\eta_{i}, \boldsymbol{r}_{T}$ and $p_{T}$ are respectively the rapidity, the sum of the transverse momenta (with respect to the beam axis) and the average scalar transverse momenta of the two final jets. In the Breit frame, where the virtual photon and target-hadron directions are back-to-back, the factorization holds when $\left|\boldsymbol{r}_{T}\right| \ll p_{T}$. The factorization of the cross-section involves the standard TMDPDFs (we have unpolarized and linearly polarized gluon TMDs, and/or quark TMDs), jet or heavy meson distributions, and a new TMD soft function built with Wilson lines aligned along the directions of the incoming hadron and the two outgoing jets. This three-direction soft function has some similarities with the one found in vector boson + jet processes in hadronic colliders [41, 42], however the structure of rapidity divergences is very different compared to the soft function discussed in those studies. The perturbative calculation of this new TMD soft function is

\footnotetext{
${ }^{1}$ In principle one may consider the case of incoming quark and outgoing gluon which then fragments into a heavy meson. However, in order to access the TMD region (small $\boldsymbol{r}_{T}$ ) this fragmentation needs to occurs near threshold, as we discuss later in the main sections, and gluon fragmentation in the kinematic end-point is power suppressed as is discussed both theoretically and phenomenologically in refs. [39, 40].
} 
performed here at one-loop using the modified $\delta$-regulator introduced in [43, 44], and we explicitly check the consistency of the factorization at the same order. For the photongluon-fusion channel, higher orders of the anomalous dimension of the new soft function can be deduced from the consistency of the anomalous dimensions of the factorized crosssection, since for the case of heavy-meson pair production all other pieces of the crosssection are also known at higher orders.

The presence of the new TMD soft function raises the question of the universality of TMDs beyond the conventional processes of Drell-Yan, semi-inclusive DIS (SIDIS) and di-hadron production in electron-positron annihilation. ${ }^{2}$ While the non-perturbative evolution is universal (in the processes we are considering here and other processes such as SIDIS), the non-perturbative corrections to the soft matrix element are not yet connected to other processes. It is therefore non-trivial to independently extract the gluon TMDPDFs. However, the size of universality breaking effects can be estimated phenomenologically by comparison with simpler processes. ${ }^{3}$ For a quantitative analysis of these effects further theoretical advancements are needed.

The paper is organized as follows: in section 2 we set the notation to be used in the rest of the paper and we give the dijet process factorization theorem. In section 3 we extend the discussion to the heavy-meson pair production and we comment on the universality of the heavy-quark jet functions that appear in our factorization theorem, and the corresponding fragmentation shape functions used to describe heavy-meson fragmentation at threshold. Finally we conclude in section 4. In appendices A and B we collected known results from the literature which we use. In appendix $\mathrm{C}$ we give a pedagogical review of some loop calculations made in this work.

\section{Dijet imbalance}

In this section we discuss the factorization of the cross-section for the dijet case in DIS within the soft-collinear effective theory (SCET). We do not give a detailed derivation of the factorization theorem, but we rather summarize the final result. We also present the NLO calculation for the new three-direction soft function and perform a consistency check of our results using the invariance of the cross-section under renormalization group evolution. The notation and kinematics that we develop here are also useful for the heavy-meson pair production presented in the subsequent section.

\subsection{Notation and kinematics}

Assuming that the direction of the beam is along the $\hat{z}$ axis it is useful to define the four-vector

$$
n^{\mu}=\frac{1}{\sqrt{2}}(1,0,0,1)
$$

\footnotetext{
${ }^{2}$ In the case of quark TMDs the conventional universality class has been recently expanded to include also semi-inclusive jet production in the Breit frame and jet-jet or hadron-jet decorrelation in lepton colliders [45-47].

${ }^{3}$ For example one may compare lepton-jet decorrelations in the laboratory frame against jet TMDs in the Breit frame.
} 
We also define a conjugate vector $\bar{n}^{\mu}$ by reversing the sign of the spacial coordinates. Thus, $n^{\mu}$ and $\bar{n}^{\mu}$ satisfy,

$$
n^{2}=\bar{n}^{2}=0, \quad \bar{n} \cdot n=1 .
$$

Using the vectors $n^{\mu}$ and $\bar{n}^{\mu}$ we can decompose any other four-vector, $p^{\mu}$, into its light-cone components,

$$
p^{\mu}=p_{+} \bar{n}^{\mu}+p_{-} n^{\mu}+p_{\perp}^{\mu}=\left(p_{+}, p_{-}, p_{\perp}\right)_{n},
$$

with

$$
p_{+}=n \cdot p, \quad p_{-}=\bar{n} \cdot p, \quad p^{2}=2 p_{+} p_{-}+p_{\perp}^{2}=2 p_{+} p_{-}-\boldsymbol{p}^{2} .
$$

where we use the notation $\boldsymbol{p} \equiv \vec{p}_{\perp}$. For the direction of the two jets we use $v_{1}$ and $v_{2}$, normalized as

$$
v_{J}^{2}=\bar{v}_{J}^{2}=0, \quad v_{J} \cdot \bar{v}_{J}=1, \quad \text { with } J=1,2,
$$

where the conjugate vectors $\bar{v}_{J}$, as above, are defined by reversing the sign of the spacial components. We define the standard Lorentz-invariants,

$$
Q^{2}=-q^{2}, \quad x=\frac{Q^{2}}{2 P \cdot q},
$$

where $q^{\mu}$ is the momentum of the virtual photon, $P^{\mu}$ is the momentum of the target hadron. In the Breit frame we have $q^{\mu}=(0,0,0, Q)$ and neglecting mass corrections we can solve for target hadron momentum,

$$
P^{\mu}=\frac{1}{2 x}(Q, 0,0,-Q) .
$$

The ratio of the longitudinal momenta of the incoming parton and the target hadron we denote with $\xi$,

$$
\xi=\frac{k^{+}}{P^{+}} .
$$

where $k^{\mu}$ the momenta of the parton incoming to the hard process. We can then express the variables $Q$ and $\xi$ in terms of the Born level kinematics using the pseudo-rapidities, $\eta_{1}$ and $\eta_{2}$, and the transverse momentum, $p_{T}$, of the two outgoing partons,

$$
Q=2 p_{T} \cosh \left(\eta_{-}\right) \exp \left(\eta_{+}\right), \quad \xi=2 x \cosh \left(\eta_{+}\right) \exp \left(-\eta_{+}\right),
$$

where

$$
\eta_{ \pm}=\frac{\eta_{1} \pm \eta_{2}}{2}
$$

In this expressions we have neglected corrections from the target hadron mass. The partonic Mandelstam variables in terms of the same quantities are,

$$
\begin{aligned}
& \hat{s}=(q+k)^{2}=+4 p_{T}^{2} \cosh ^{2}\left(\eta_{-}\right), \\
& \hat{t}=\left(q-p_{2}\right)^{2}=-4 p_{T}^{2} \cosh \left(\eta_{-}\right) \cosh \left(\eta_{+}\right) \exp \left(\eta_{1}\right), \\
& \hat{u}=\left(q-p_{1}\right)^{2}=-4 p_{T}^{2} \cosh \left(\eta_{-}\right) \cosh \left(\eta_{+}\right) \exp \left(\eta_{2}\right),
\end{aligned}
$$


where $p_{1}^{\mu}$ and $p_{2}^{\mu}$ are the momenta of the outgoing partons. It is easy to check that the partonic Mandelstam variables satisfy,

$$
\hat{s}+\hat{t}+\hat{u}=-Q^{2} .
$$

We denote the transverse momentum imbalance of the two jets with $\boldsymbol{r}_{T}$, where the hard transverse momentum $p_{T}$ corresponds, up-to power corrections, to the average transverse momenta of the two jets,

$$
\boldsymbol{r}_{T}=\boldsymbol{p}_{1 T}+\boldsymbol{p}_{2 T}, \quad p_{T}=\frac{\left|\boldsymbol{p}_{1 T}\right|+\left|\boldsymbol{p}_{2 T}\right|}{2}
$$

where the sub-index 1,2 refers to the final jets. At Born level $\boldsymbol{p}_{1 T}=-\boldsymbol{p}_{2 T}$ and thus $\boldsymbol{r}_{T}=0$. However, hadronization of the outgoing partons will form jet-like configurations along similar directions and wide angle radiation could escape the jet clustering algorithm, which will then contribute to the imbalance.

We now consider the kinematic region in which the factorization theorem holds in comparison to the coverage of EIC. We first evaluate the constraints on the rapidities of the two jets, $\eta_{1}$ and $\eta_{2}$. To do so we require that $\hat{s} \sim|\hat{t}| \sim|\hat{u}|$ and quantitatively we implement that by imposing,

$$
\frac{1}{j}<\frac{\hat{s}}{|\hat{t}|}, \frac{\hat{s}}{|\hat{u}|}, \frac{|\hat{u}|}{|\hat{t}|}<j
$$

This constrains the values of rapidities within the blue region as illustrated in figure 2 for the cases $j=2$ (green-dashed) and $j=4$ (blue-solid). In addition to avoid contributions from the resolved photon processes we require $Q \sim p_{T}$ which quantitatively we implement by imposing,

$$
\frac{1}{k}<\frac{Q^{2}}{4 p_{T}^{2}}<k
$$

The relevant region in the two jet rapidities is shown as the red-dotted and magentadotdashed shaded areas for $k=4$ and $k=2$ respectively in figure 2. The overlap of the regions constraint by (2.15) and (2.14) gives the allowed values of rapidites for which the factorization theorem holds and contamination from resolved photo-production processes is minimal. This suggests that the two processes we are considering here, are described by two clearly separated jets (or heavy mesons) within the central rapidity region. As expected, when tighten the constraint (by decreasing the values of $j$ and/or $k$ ) the relevant region shrinks around the central point, $\eta_{1}=\eta_{2}=0$.

Constrained within the overlapping region of rapidities in figure 2 and for $p_{T} \in$ $[4,20] \mathrm{GeV}$ and $\xi \in\left[10^{-2}, 1\right]$ we construct the $\left(x, Q^{2}\right)$ values relevant for the process we are considering. We show the corresponding region in $\left(x, Q^{2}\right)$ in figure 3 for $j=k=2$ (green-dashed) and $j=k=4$ (blue-solid). We also included (brown-shaded area) the expected $\left(x, Q^{2}\right)$ coverage at EIC for three different center of mass energies: $\sqrt{s}=140,63$, and $28 \mathrm{GeV}$. We see that for all three energies there is significant overlap of the investigated process and the EIC coverage, but the overlapping region increases at higher beam energies. At the same time the cases $j=k=2$ and $j=k=4$ does seem to change the overlapping region with the EIC coverage in the small $Q^{2}$ region. Although the change 


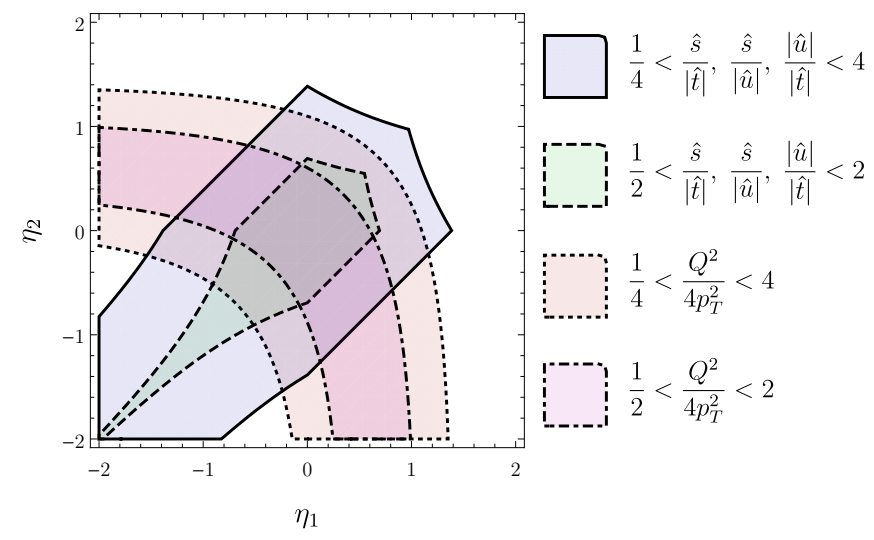

Figure 2. The jet-rapidity regions for which $\hat{s} \sim|\hat{t}| \sim|\hat{u}|:$ blue-solid $(j=4)$ and green-dashed $(j=2)$ and $Q \sim p_{T}$ : red-dotted $k=4$ and magenta-dotdashed $(k=2)$. The overlapping region which is found at central rapidities is where we anticipated small contributions to the cross section beyond factorization.

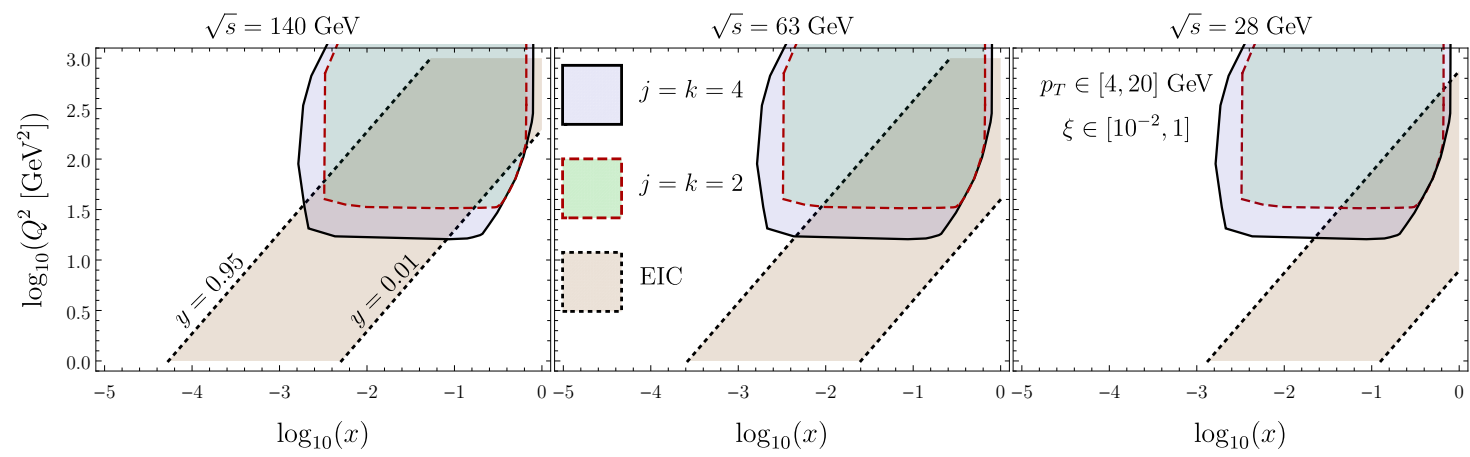

Figure 3. The $\left(x, Q^{2}\right)$ coverage of EIC (brown/dashed) compared to the di-jet events with $j=k=4$ factorization regime (blue-solid) and with $j=k=2$ (green-dashed). The factorization regime has been constructed assuming $p_{T} \in[4,20] \mathrm{GeV}, \xi \in\left[10^{-2}, 1\right]$, and jet rapidities in the overlapping region in figure 2 .

of the overlapping region is not large, the lost region is statistically important. A further investigation into this using monte carlo event generators will be important in order to determine the kinematic constraints on the jets, for which a reasonable compromise between statistics and factorization-corrections can be made. ${ }^{4}$

\subsection{Factorization theorem for dijet production}

There are two channels for the dijet process that we need to consider: a) the gluon-photon fusion channel which corresponds to the partonic process, $\gamma^{*} g \rightarrow f \bar{f}$, and b) the incoming quark or antiquark channel from the partonic process, $\gamma^{*} f \rightarrow g f$. The factorization that we

\footnotetext{
${ }^{4}$ Note that here for both scenarios we consider $j=k$ even-though $j$ and $k$ are independent variables and maybe chosen to be not equal. They need to be however, order $\mathcal{O}(1)$ numbers. For example, the case $j=2, k=4$, which is not shown here, seems to have very little effect on the overlapping region with the EIC coverage, compared to the case $j=4, k=4$.
} 
propose holds when $\left|\boldsymbol{r}_{T}\right| \ll p_{T}$ and we treat the cross-sections only at leading power in an $\left|\boldsymbol{r}_{T}\right| / p_{T}$ or $\left|\boldsymbol{r}_{T}\right| / Q$ expansion. Within this constraint, the gluon-photon channel factorized cross-section is

$$
\begin{aligned}
\frac{d \sigma\left(\gamma^{*} g\right)}{d x d \eta_{1} d \eta_{2} d p_{T} d \boldsymbol{r}_{T}}= & \sum_{f} H_{\gamma^{*} g \rightarrow f \bar{f}}^{\mu \nu}(\hat{s}, \hat{t}, \hat{u}, \mu) \int \frac{d^{2} \boldsymbol{b}}{(2 \pi)^{2}} \exp \left(i \boldsymbol{b} \cdot \boldsymbol{r}_{T}\right) F_{g, \mu \nu}\left(\xi, \boldsymbol{b}, \mu, \zeta_{1}\right) \\
& \times S_{\gamma g}\left(\boldsymbol{b}, \eta_{1}, \eta_{2}, \mu, \zeta_{2}\right)\left(\mathcal{C}_{f}(\boldsymbol{b}, R, \mu) J_{f}\left(p_{T}, R, \mu\right)\right)\left(\mathcal{C}_{\bar{f}}(\boldsymbol{b}, R, \mu) J_{\bar{f}}\left(p_{T}, R, \mu\right)\right),
\end{aligned}
$$

where the sum runs over all light quark and antiquark flavours $f$. Here we consider jets with their momentum reconstructed with the so called E-scheme, that is, the momentum of the jets is given by the sum of all jet-constituents. For these jets and for small jet radius $(R \ll 1)$ the cross-section can be factorized in terms of the collinear-soft function $\mathcal{C}_{i}(\boldsymbol{b}, R)$, that describes the soft radiation close to the jet boundary and the exclusive jet functions $J_{i}$, that describe the collinear and energetic radiation confined within the jet. ${ }^{5}$ These functions are calculated up to NLO for generic $k_{T}$-type and cone jet algorithms in [41, 49]. The corresponding operator definitions are given in the appendix A. In addition, the factorization theorem contains the dijet soft function $S_{\gamma g}$, which is discussed and calculated at one-loop in next section. Finally, we have the gluon TMD function $F_{g}$, whose operator definition can also be found in appendix A.

We notice at this point that the latter two functions, $S_{\gamma g}$ and $F_{g}$ in (2.16), have an intricate interplay due to the rapidity divergences, which introduces the rapidity scale dependence, $\zeta_{1,2}$, in the corresponding functions. In section 2.4 we explain this issue and the role played by the zero-bin subtractions, which lead to the proper definition of the dijet soft function and the TMDs.

The gluon TMD for an unpolarized proton can be further separated into two pieces, the unpolarized gluon distribution $f_{1}(\xi, \boldsymbol{b})$ and the linearly polarized gluon contribution $h_{1}^{\perp}(\xi, \boldsymbol{b})$ :

$$
F_{g}^{\mu \nu}(\xi, \boldsymbol{b})=f_{1}(\xi, \boldsymbol{b}) \frac{g_{T}^{\mu \nu}}{d-2}+h_{1}^{\perp}(\xi, \boldsymbol{b})\left(\frac{g_{T}^{\mu \nu}}{d-2}+\frac{b^{\mu} b^{\nu}}{\boldsymbol{b}^{2}}\right)
$$

with $g_{T}^{\mu \nu}=g^{\mu \nu}-n^{\mu} \bar{n}^{\nu}-\bar{n}^{\mu} n^{\nu}$. Both of these functions are known perturbatively up to nextto-next-to leading order (NNLO) $[5,44,50]$. The evolution of the TMDs, which is universal (see e.g. [51, 52]), is also known up to $\mathrm{N}^{3} \mathrm{LO}[43,53,54]$. In $(2.17)$ we have included only twist-2 TMDs, neglecting higher twists in the TMD expansion. This is sufficient since we consider higher-twist functions suppressed, which is consistent with SIDIS studies as in [55]. However, we have no quantitative estimate of these functions and further investigation is important. The inclusion of the higher-twist contributions is beyond the scope of this work and we leave such considerations for future studies. The hard function is then decomposed in two tensor structures:

$$
H_{\gamma^{*} g \rightarrow f \bar{f}}^{\mu \nu}=\sigma_{0}^{g U} H_{\gamma^{*} g \rightarrow f \bar{f}}^{U} \frac{g_{T}^{\mu \nu}}{d-2}+\sigma_{0}^{g L} H_{\gamma^{*} g \rightarrow f \bar{f}}^{L}\left(-\frac{g_{T}^{\mu \nu}}{d-2}+\frac{v_{1 T}^{\mu} v_{2 T}^{\nu}+v_{2 T}^{\mu} v_{1 T}^{\nu}}{2 v_{1 T} \cdot v_{2 T}}\right),
$$

\footnotetext{
${ }^{5}$ For recent developments on jet algorithms for DIS see ref. [48]. We also plan in the near future to complete the NLO calculation of jet functions in central rapidity regions for the Centauro algorithm.
} 
where we have ignored all terms proportional to the four-vector $n^{\mu}$ (since they vanish after Lorentz-contraction with the gluon beam function) and any anti-symmetric combinations (since the cross-section is integrated over angles). The coefficients $\sigma_{0}^{g U(L)}$ are introduced such that the leading order hard functions are normalized to the unity, i.e. $H_{\mathrm{LO}}^{U(L)}=1+$ $\mathcal{O}\left(\alpha_{s}\right)$. With this we can now separate the cross-section into a contribution from the unpolarized gluons and one from the linearly polarized gluons. We write the cross-section as

$$
d \sigma\left(\gamma^{*} g\right)=d \sigma^{U}\left(\gamma^{*} g\right)+d \sigma^{L}\left(\gamma^{*} g\right),
$$

where

$$
\begin{aligned}
\frac{d \sigma^{U}\left(\gamma^{*} g\right)}{d x d \eta_{1} d \eta_{2} d p_{T} d \boldsymbol{r}_{T}}= & \sigma_{0}^{g U} \sum_{f} H_{\gamma^{*} g \rightarrow f \bar{f}}^{U}(\hat{s}, \hat{t}, \hat{u}, \mu) \int \frac{d^{2} \boldsymbol{b}}{(2 \pi)^{2}} \exp \left(i \boldsymbol{b} \cdot \boldsymbol{r}_{T}\right) f_{1}\left(\xi, \boldsymbol{b}, \mu, \zeta_{1}\right) \\
& \times S_{\gamma g}\left(\boldsymbol{b}, \zeta_{2}, \mu\right)\left(\mathcal{C}_{f}(\boldsymbol{b}, R, \mu) J_{f}\left(p_{T}, R, \mu\right)\right)\left(\mathcal{C}_{\bar{f}}(\boldsymbol{b}, R, \mu) J_{\bar{f}}\left(p_{T}, R, \mu\right)\right)
\end{aligned}
$$

and

$$
\begin{aligned}
\frac{d \sigma^{L}\left(\gamma^{*} g\right)}{d x d \eta_{1} d \eta_{2} d p_{T} d \boldsymbol{r}_{T}} & =\sigma_{0}^{g L} \sum_{f} H_{\gamma^{*} g \rightarrow f \bar{f}}^{L}(\hat{s}, \hat{t}, \hat{u}, \mu) \int \frac{d^{2} \boldsymbol{b}}{(2 \pi)^{2}} \exp \left(i \boldsymbol{b} \cdot \boldsymbol{r}_{T}\right) h_{1}^{\perp}\left(\xi, \boldsymbol{b}, \mu, \zeta_{1}\right) \\
\times & \frac{s_{\boldsymbol{b}}^{2}-c_{\boldsymbol{b}}^{2}}{2} S_{\gamma g}\left(\boldsymbol{b}, \zeta_{2}, \mu\right)\left(\mathcal{C}_{f}(\boldsymbol{b}, R, \mu) J_{f}\left(p_{T}, R, \mu\right)\right)\left(\mathcal{C}_{\bar{f}}(\boldsymbol{b}, R, \mu) J_{\bar{f}}\left(p_{T}, R, \mu\right)\right) .
\end{aligned}
$$

We used the shorthand notation $s_{\boldsymbol{b}}$ and $c_{\boldsymbol{b}}$ for the sine and cosine of the angle between the vectors $\boldsymbol{b}$ and $\boldsymbol{v}_{1 T}$, respectively. The hard factors are calculated up to NNLO in the unpolarized case in $[56,57]$, while for the linearly polarized gluons they are calculated at LO in [58]. All hard coefficients are reported in appendix A.

Finally, the incoming quark channel has contributions only from the unpolarized gluon jets and the cross-section is given by the following factorized formula:

$$
\begin{aligned}
\frac{d \sigma^{U}\left(\gamma^{*} f\right)}{d x d \eta_{1} d \eta_{2} d p_{T} d \boldsymbol{r}_{T}}= & \sigma_{0}^{f U} \sum_{f} H_{\gamma^{*} f \rightarrow g f}^{U}(\hat{s}, \hat{t}, \hat{u}, \mu) \int \frac{d^{2} \boldsymbol{b}}{(2 \pi)^{2}} \exp \left(i \boldsymbol{b} \cdot \boldsymbol{r}_{T}\right) F_{f}\left(\xi, \boldsymbol{b}, \mu, \zeta_{1}\right) \\
& \times S_{\gamma f}\left(\boldsymbol{b}, \zeta_{2}, \mu\right)\left(\mathcal{C}_{g}(\boldsymbol{b}, R, \mu) J_{g}\left(p_{T}, R, \mu\right)\right)\left(\mathcal{C}_{f}(\boldsymbol{b}, R, \mu) J_{f}\left(p_{T}, R, \mu\right)\right),
\end{aligned}
$$

where the sum runs over quarks and anti-quarks and $F_{f}$ is the $f$-flavor quark/antiquark unpolarized TMDPDF.

\subsection{The dijet soft function at NLO}

The only new matrix element in the previous section is the soft function. Here we give the operator matrix element definition of the soft function and we proceed with the NLO calculation. The details of the calculation are collected in the appendix. We start defining the soft function for the photon-gluon fusion process:

$$
\begin{aligned}
\hat{S}_{\gamma g}(\boldsymbol{b})= & \frac{1}{C_{F} C_{A}}\langle 0| \mathcal{S}_{n}^{\dagger}(\boldsymbol{b},-\infty)_{c a^{\prime}} \operatorname{Tr}\left[S_{v_{2}}(+\infty, \boldsymbol{b}) T^{a^{\prime}} S_{v_{1}}^{\dagger}(+\infty, \boldsymbol{b})\right. \\
& \left.\times S_{v_{1}}(+\infty, 0) T^{a} S_{v_{2}}^{\dagger}(+\infty, 0)\right] \mathcal{S}_{n}(0,-\infty)_{a c}|0\rangle
\end{aligned}
$$


The soft function corresponding to the case of incoming quark or antiquark is obtained with the exchange

$$
\hat{S}_{\gamma f}=\hat{S}_{\gamma g}\left(n \leftrightarrow v_{2}\right)
$$

The Wilson lines are defined as

$$
\begin{aligned}
S_{v}(+\infty, \xi) & =P \exp \left[-i g \int_{0}^{+\infty} d \lambda v \cdot A(\lambda v+\xi)\right], \\
S_{\bar{v}}^{\dagger}(+\infty, \xi) & =P \exp \left[i g \int_{0}^{+\infty} d \lambda \bar{v} \cdot A(\lambda \bar{v}+\xi)\right], \\
S_{n}(+\infty, \xi) & =\lim _{\delta^{+} \rightarrow 0} P \exp \left[-i g \int_{0}^{+\infty} d \lambda n \cdot A(\lambda n+\xi) e^{-\delta^{+} \lambda}\right] .
\end{aligned}
$$

We omit here $T$-Wilson lines which are needed in singular gauges. We also distinguish Wilson lines in the adjoint and fundamental SU $\left(N_{c}\right)$ representations using $\mathcal{S}$ and $S$ respectively. Note that the $\delta$-regulator is introduced only in $S_{n}\left(\mathcal{S}_{n}\right)$. We write the soft function as a series in $a_{s}=\alpha_{s} /(4 \pi)$

$$
\hat{S}=\sum_{m=0}^{\infty} a_{s}^{m} \hat{S}^{[m]} .
$$

At tree level $\hat{S}^{[0]}=1$, and at one-loop only the diagrams with a real gluon give a non-zero result. We can therefore write the one-loop soft function as a sum of diagrams with one real gluon exchange between the three soft Wilson lines,

$$
\hat{S}^{[1]}=\frac{1}{2} \sum_{\substack{i \neq j \\ j \in\{1,2, B\}}} C^{i j} \hat{S}_{i j}^{[1]},
$$

where the suffix indicates the Wilson lines connected by the exchanged gluon (1 and 2 for the two jets and $B$ for the beam), including the mirror diagrams as well (note that $C^{i j}=C^{j i}$ and $\hat{S}_{i j}^{[1]}=\hat{S}_{j i}^{[1]}$ ). Notice that the mirror diagrams for $\hat{S}_{J B}$ will introduce an additional $i \pi$ component, as discussed in more detail in the appendix. The coefficients $C^{i j}$ are the color factors and they are different for the two channels $\gamma^{*} g$ and $\gamma^{*} f$,

$$
\begin{aligned}
C_{\gamma g}^{1 B} & =C_{\gamma g}^{2 B}=C_{A}, & C_{\gamma g}^{12} & =2 C_{F}-C_{A}, \\
C_{\gamma f}^{2 B} & =C_{\gamma f}^{12}=C_{A}, & C_{\gamma f}^{1 B} & =2 C_{F}-C_{A} .
\end{aligned}
$$

The relevant diagrams are shown in figure 4 and the corresponding contributions are

$$
\hat{S}_{J B}^{[1]}=\pi(4 \pi)^{2} \mu^{2 \epsilon}\left(n \cdot v_{J}\right) I_{J B}+\text { mir. diag. }, \quad \hat{S}_{12}^{[1]}=\pi(4 \pi)^{2} \mu^{2 \epsilon}\left(v_{1} \cdot v_{2}\right) I_{12}+\text { mir. diag. },
$$

where $J=1,2$ and the corresponding integrals are

$$
I_{J B}=\int \frac{d^{d} k}{(2 \pi)^{d}} \frac{e^{i \boldsymbol{k} \cdot \boldsymbol{b}} \delta\left(k^{2}\right) \theta\left(k_{+}\right)}{\left(n \cdot k+i \delta^{+}\right)\left(v_{J} \cdot k\right)}, \quad I_{12}=\int \frac{d^{d} k}{(2 \pi)^{d}} \frac{e^{i \boldsymbol{k} \cdot \boldsymbol{b}} \delta\left(k^{2}\right) \theta\left(k_{+}\right)}{\left(v_{1} \cdot k\right)\left(v_{2} \cdot k\right)}
$$




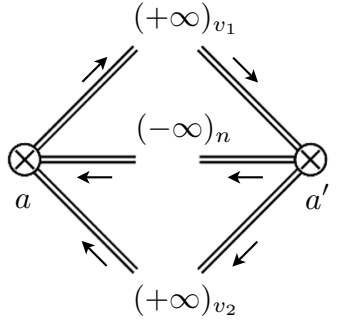

(a)

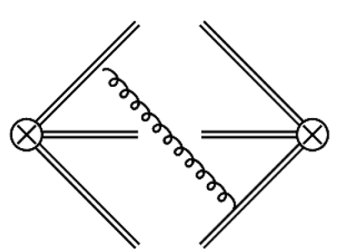

(b)

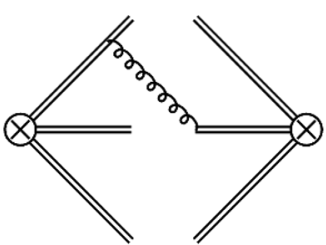

(c)

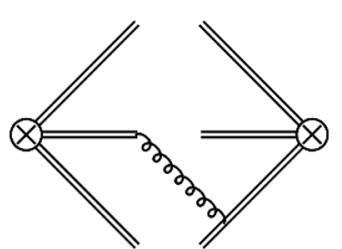

(d)

Figure 4. Tree level soft function is shown in diagram (a). Each double line represents a Wilson line whose pointing direction is also reported as $( \pm \infty)_{u}, u=n, v, \bar{v} ; a$ and $a^{\prime}$ are color indices. Diagrams (b), (c) and (d) also contribute to the NLO soft function. Virtual contributions vanish and therefore they are not shown here. Mirror diagrams are also not shown.

with $d=4-2 \epsilon$. The results at all orders in $\epsilon$ are

$$
\hat{S}_{J B}^{[1]}=-2 \frac{\mu^{2 \epsilon} B^{\epsilon}}{(4 \pi)^{-\epsilon}} \Gamma(-\epsilon)\left[\ln \left(-\frac{i\left(\boldsymbol{v}_{J} \cdot \boldsymbol{b}\right) \delta^{+}}{n \cdot v_{J}}\right)+\gamma_{E}\right],
$$

and

$$
\hat{S}_{12}^{[1]}=2 \frac{\mu^{2 \epsilon} B^{\epsilon}}{(4 \pi)^{-\epsilon}} \Gamma(-\epsilon)\left[\Gamma(-\epsilon) \Gamma(1+\epsilon)\left(\frac{1+A_{\boldsymbol{b}}}{-A_{\boldsymbol{b}}}\right)^{\epsilon}-\frac{\Gamma(-1-\epsilon)}{\Gamma(-\epsilon)} A_{\boldsymbol{b} 2} F_{1}\left(1,1,2+\epsilon,-A_{\boldsymbol{b}}\right)\right],
$$

with the shorthand notation,

$$
A_{\boldsymbol{b}}=\frac{\left(v_{1} \cdot v_{2}\right)}{2\left(v_{1} \cdot \hat{b}\right)\left(v_{2} \cdot \hat{b}\right)}=-\frac{\hat{s}}{4 p_{T}^{2} c_{b}^{2}}, \quad B=\frac{|\boldsymbol{b}|^{2}}{4},
$$

and $\hat{b}=(0,0, \boldsymbol{b}) /|\boldsymbol{b}|$. Note that $A_{\boldsymbol{b}}$ is a function of the angle $\phi_{b J}$ only and it does not depend on $|\boldsymbol{b}| . A_{\boldsymbol{b}}$ is also dimensionless, longitudinal boost invariant, and it is bounded to negative values less than -1 , i.e., $A_{\boldsymbol{b}} \leq-1$. The function ${ }_{2} F_{1}$ is the standard hypergeometric function and the $\epsilon$ expansion for this function can be written as follows

$$
{ }_{2} F_{1}(1,1,2+\epsilon,-z)=\frac{1}{z} \ln (1+z)+\frac{\epsilon}{z}\left[-\frac{\pi^{2}}{6}+\left(1+\ln \left(\frac{1+z}{z}\right)\right) \ln (1+z)+\operatorname{Li}_{2}\left(\frac{1}{1+z}\right)\right]+\mathcal{O}\left(\epsilon^{2}\right) .
$$

Adding all contributions and expanding in $\epsilon$ we obtain the bare soft function. For the $\gamma^{*} g$-channel we have:

$$
\begin{aligned}
\hat{S}_{\gamma g}^{\text {bare }}(\boldsymbol{b})= & \hat{S}_{\gamma g}^{\text {finite }}(\boldsymbol{b})+a_{s}\left\{C_{A}\left[-\frac{2}{\epsilon^{2}}+\frac{2}{\epsilon}\left(2 \ln \left(\frac{\sqrt{2} \delta^{+}}{\mu}\right)+\ln \left(2 A_{n}\right)\right)\right]\right. \\
& \left.+2 C_{F}\left[\frac{2}{\epsilon^{2}}+\frac{2}{\epsilon} \ln \left(\frac{B \mu^{2} e^{2 \gamma_{E}}}{-A_{\boldsymbol{b}}}\right)\right]\right\},
\end{aligned}
$$

for the $\gamma^{*} f$-channel:

$$
\begin{aligned}
\hat{S}_{\gamma f}^{\text {bare }}(\boldsymbol{b})= & \hat{S}_{\gamma f}^{\text {finite }}(\boldsymbol{b})+a_{s}\left\{C_{A}\left[\frac{2}{\epsilon^{2}}+\frac{2}{\epsilon}\left(\ln \frac{\left(n \cdot v_{1}\right)\left(\boldsymbol{v}_{2} \cdot \boldsymbol{b}\right)}{\left(n \cdot v_{2}\right)\left(\boldsymbol{v}_{1} \cdot \boldsymbol{b}\right)}+\ln \left(\frac{B \mu^{2} e^{2 \gamma_{E}}}{-A_{\boldsymbol{b}}}\right)\right)\right]\right. \\
& \left.+\frac{4}{\epsilon} C_{F} \ln \left(-\frac{i \boldsymbol{v}_{\mathbf{1}} \cdot \boldsymbol{b} \delta^{+} e^{\gamma_{E}}}{n \cdot v_{1}}\right)\right\},
\end{aligned}
$$


where the finite part of the soft function is

$$
\begin{aligned}
\hat{S}_{\gamma g}^{\text {finite }}(\boldsymbol{b})= & 1+a_{s}\left\{C _ { A } \left[\ln \left(B \mu^{2} e^{2 \gamma_{E}}\right)\left(\ln \left(B \mu^{2} e^{2 \gamma_{E}}\right)+4 \ln \left(\frac{\sqrt{2} \delta^{+}}{\mu}\right)+2 \ln \left(2 A_{n}\right)\right)-\ln ^{2}\left(-A_{\boldsymbol{b}}\right)\right.\right. \\
& \left.\left.-\frac{\pi^{2}}{6}-2 \operatorname{Li}_{2}\left(1+A_{\boldsymbol{b}}\right)\right]+C_{F}\left[\frac{\pi^{2}}{3}+2 \ln ^{2}\left(\frac{B \mu^{2} e^{2 \gamma_{E}}}{-A_{\boldsymbol{b}}}\right)+4 \operatorname{Li}_{2}\left(1+A_{\boldsymbol{b}}\right)\right]\right\}, \quad(2.37)
\end{aligned}
$$

and

$$
\begin{aligned}
\hat{S}_{\gamma f}^{\text {finite }}(\boldsymbol{b})= & 1+a_{s}\left\{C _ { A } \left[\frac{\pi^{2}}{6}+\ln ^{2}\left(\frac{B \mu^{2} e^{2 \gamma_{E}}}{-A_{\boldsymbol{b}}}\right)+2 \operatorname{Li}_{2}\left(1+A_{\boldsymbol{b}}\right)\right.\right. \\
& \left.+2 \ln \left(B \mu^{2} e^{2 \gamma_{E}}\right) \ln \frac{\left(n \cdot v_{1}\right)\left(\boldsymbol{v}_{2} \cdot \boldsymbol{b}\right)}{\left(n \cdot v_{2}\right)\left(\boldsymbol{v}_{1} \cdot \boldsymbol{b}\right)}\right] \\
& \left.+4 C_{F} \ln \left(B \mu^{2} e^{2 \gamma_{E}}\right) \ln \left(-\frac{i \boldsymbol{v}_{\mathbf{1}} \cdot \boldsymbol{b} \delta^{+} e^{\gamma_{E}}}{n \cdot v_{1}}\right)\right\} .
\end{aligned}
$$

To simplify the results we have used the notation

$$
A_{n}=\frac{\left(v_{1} \cdot v_{2}\right)}{2\left(v_{1} \cdot n\right)\left(v_{2} \cdot n\right)} .
$$

\subsection{The zero-bin subtraction and the universal TMDs}

Here we reorganize the factorization theorem such that the cross-section is expressed in terms of rapidity divergence-free TMDs as presented in the factorization theorem in (2.20), (2.21) and (2.22). In this way we make clear the dependence on the universal TMDPDFs and the unknown TMD soft function. To do this we write the TMD beam function in terms of the zero-bin un-subtracted term divided by the back-to-back soft function,

$$
\hat{B}_{i}\left(\xi, \boldsymbol{b}, \mu, k^{-} \delta_{+}\right)=\frac{B_{i}^{\mathrm{un}} \cdot\left(\xi, \boldsymbol{b}, \mu, k^{-} / \delta^{-}\right)}{S\left(\boldsymbol{b}, \mu, \sqrt{\delta^{+} \delta^{-}}\right)}
$$

The back-to-back two-direction soft function operator definition can be found in appendix A. Then following $[43,59]$ we can factorize the soft function as

$$
S\left(\boldsymbol{b}, \mu, \sqrt{\delta^{+} \delta^{-}}\right)=S^{\frac{1}{2}}\left(\boldsymbol{b}, \mu, \delta^{+} \nu\right) S^{\frac{1}{2}}\left(\boldsymbol{b}, \mu, \delta^{-} / \nu\right),
$$

where $\nu$ is an arbitrary positive number which plays a bookkeeping role and will be removed from the final result, introducing this way a constraint on the product of rapidity scales. The bare function $\left(S^{\text {bare }}\right)^{\frac{1}{2}}$ is

$$
\begin{aligned}
\left(S_{i}^{\text {bare }}(\boldsymbol{b}, \delta)\right)^{\frac{1}{2}}= & 1+a_{s} C_{i}\left\{-\frac{2}{\epsilon^{2}}+\frac{4}{\epsilon} \ln \left(\frac{\sqrt{2} \delta}{\mu}\right)\right. \\
& \left.+\ln \left(B \mu^{2} e^{2 \gamma_{E}}\right)\left[4 \ln \left(\frac{\sqrt{2} \delta}{\mu}\right)+\ln \left(B \mu^{2} e^{2 \gamma_{E}}\right)\right]+\frac{\pi^{2}}{6}\right\}+\mathcal{O}\left(a_{s}^{2}\right),
\end{aligned}
$$

where here and in the rest of this manuscript we used the shorthand notation

$$
\gamma_{g}=\frac{\beta_{0}}{2 C_{A}}, \quad \gamma_{q}=\frac{3}{2}, \quad C_{f}=C_{F}=\frac{N_{c}^{2}-1}{2 N_{c}}, \quad C_{g}=C_{A}=N_{c} .
$$


Thus we can now reorganize the beam and soft function matrix elements (denoted by the "hat" notation) into a product of TMDs as they appear in the factorization theorem,

$$
\hat{B}_{i}\left(\xi, \boldsymbol{b}, \mu, k^{-} \delta_{+}\right) \hat{S}_{\gamma i}\left(\boldsymbol{b}, \mu, \sqrt{A_{n}} \delta_{+}\right)=F_{i}\left(\xi, \boldsymbol{b}, \mu, \zeta_{1}\right) S_{\gamma i}\left(\boldsymbol{b}, \mu, \zeta_{2}\right)
$$

where the functions in the r.h.s. of (2.44) are respectively

$$
F_{i}\left(\xi, \boldsymbol{b}, \mu, \zeta_{1}\right)=\left.\frac{B_{i}^{\mathrm{un} .}\left(\xi, \boldsymbol{b}, \mu, k^{-} / \delta^{-}\right)}{S^{\frac{1}{2}}\left(\boldsymbol{b}, \mu, \delta^{-} / \nu\right)}\right|_{\sqrt{2} k^{-} / \nu \rightarrow \sqrt{\zeta_{1}}},
$$

that is, the universal TMDPDF as defined in other observables such as Drell-Yan and semi-inclusive DIS and

$$
S_{\gamma i}\left(\boldsymbol{b}, \mu, \zeta_{2}\right)=\left.\frac{\hat{S}_{\gamma i}\left(\boldsymbol{b}, \mu, \sqrt{A_{n}} \delta^{+}\right)}{S^{\frac{1}{2}}\left(\boldsymbol{b}, \mu, \delta^{+} \nu\right)}\right|_{\nu / \sqrt{2 A_{n}} \rightarrow \sqrt{\zeta_{2}}},
$$

that is the unknown soft function now incorporated in a rapidity divergent-free ratio. We can thus eliminate the dependence on the arbitrary parameter $\nu$ by introducing the following constraint

$$
\zeta_{1} \zeta_{2}=\frac{\left(k^{-}\right)^{2}}{A_{n}}=\frac{\hat{u} \hat{t}}{\hat{s}}
$$

where $\hat{s}, \hat{t}$, and $\hat{u}$ are the partonic Mandelstam variables and thus the combination $\zeta_{1} \zeta_{2}$ is Lorentz invariant and in the Breit frame, or any other frame boosted along the proton direction, we have: $\zeta_{1} \zeta_{2}=p_{T}^{2}$. Notice that the procedure to obtain (2.47) is totally analogous to the one used in Drell-Yan or SIDIS [60,61], TMD factorization theorem. In that case we have that $\zeta_{1,2}$ have both a square mass dimension and $\zeta_{1} \zeta_{2}=Q^{4}$, while in the present case $\zeta_{2}$ is dimensionless quantity but $\zeta_{1}$, as usual, has dimensions of mass squared. The natural way to choose the values of $\zeta_{1}$ and $\zeta_{2}$ is

$$
\zeta_{1}=p_{T}^{2}, \quad \zeta_{2}=1
$$

This way we have the standard evolution for the TMDPDF up to the hard scale and the ratio of soft functions in (2.46) has no large rapidity logarithms and thus does not require evolution in rapidity.

The renormalized soft function can then be written in terms of the renormalization kernel $Z^{S}$ and the bare soft function which is simply the ratio of the bare functions that appear in (2.46),

$$
S_{\gamma i}^{\mathrm{bare}}\left(\boldsymbol{b}, \zeta_{2}\right)=Z_{\gamma i}^{S}\left(\boldsymbol{b}, \mu, \zeta_{2}\right) S_{\gamma i}\left(\boldsymbol{b}, \mu, \zeta_{2}\right)
$$

In the $\overline{\mathrm{MS}}$ scheme for the $\left(\gamma^{*} g\right)$ channel we have

$$
\begin{aligned}
S_{\gamma g}\left(\boldsymbol{b}, \mu, \zeta_{2}\right)= & 1+a_{s}\left\{C_{F}\left[\frac{\pi^{2}}{3}+2 \ln ^{2}\left(\frac{B \mu^{2} e^{2 \gamma_{E}}}{-A_{\boldsymbol{b}}}\right)+4 \operatorname{Li}_{2}\left(1+A_{\boldsymbol{b}}\right)\right]\right. \\
& \left.+C_{A}\left[-2 \ln \left(B \mu^{2} e^{2 \gamma_{E}}\right) \ln \zeta_{2}-\ln ^{2}\left(-A_{\boldsymbol{b}}\right)-\frac{\pi^{2}}{3}-2 \operatorname{Li}_{2}\left(1+A_{\boldsymbol{b}}\right)\right]\right\}+\mathcal{O}\left(a_{s}^{2}\right),
\end{aligned}
$$


and for the $\left(\gamma^{*} f\right)$ channel we have

$$
\begin{aligned}
S_{\gamma f}\left(\boldsymbol{b}, \mu, \zeta_{2}\right)= & 1+a_{s}\left\{c C _ { A } \left[\frac{\pi^{2}}{6}+\ln ^{2}\left(\frac{B \mu^{2} e^{2 \gamma_{E}}}{-A_{\boldsymbol{b}}}\right)+2 \operatorname{Li}_{2}\left(1+A_{\boldsymbol{b}}\right)\right.\right. \\
& \left.+2 \ln \left(B \mu^{2} e^{2 \gamma_{E}}\right) \ln \frac{\left(n \cdot v_{1}\right)\left(\boldsymbol{v}_{2} \cdot \boldsymbol{b}\right)}{\left(n \cdot v_{2}\right)\left(\boldsymbol{v}_{1} \cdot \boldsymbol{b}\right)}\right] \\
& +C_{F} \ln \left(B \mu^{2} e^{2 \gamma_{E}}\right)\left[\ln \left(B \mu^{2} e^{2 \gamma_{E}}\right)-2 \ln \zeta_{2}+2 \ln \left(\frac{2\left(n \cdot v_{2}\right)}{\left(v_{1} \cdot v_{2}\right)\left(n \cdot v_{2}\right)}\right)\right. \\
& \left.\left.-\frac{\pi^{2}}{6}+4 \ln \left(-i \boldsymbol{v}_{1} \cdot \hat{\boldsymbol{b}}\right)\right]\right\}+\mathcal{O}\left(a_{s}^{2}\right),
\end{aligned}
$$

Note that the imaginary terms cancel in the sum and after taking the Fourier transform in momentum space, resulting in a real cross-section, which we have checked explicitly up to the NLO contributions. The corresponding renormalization functions are

$$
Z_{\gamma g}^{S}\left(\boldsymbol{b}, \mu, \zeta_{2}\right)=1+a_{s}\left\{C_{F}\left[\frac{4}{\epsilon^{2}}+\frac{4}{\epsilon} \ln \left(\frac{B \mu^{2} e^{2 \gamma_{E}}}{-A_{\boldsymbol{b}}}\right)\right]-C_{A} \frac{2}{\epsilon} \ln \zeta_{2}\right\}+\mathcal{O}\left(a_{s}^{2}\right),
$$

and

$$
\begin{aligned}
Z_{\gamma f}^{S}\left(\boldsymbol{b}, \mu, \zeta_{2}\right)= & 1+a_{s}\left\{C_{A}\left[\frac{2}{\epsilon^{2}}-\frac{2}{\epsilon}\left(\ln \frac{\left(n \cdot v_{1}\right)\left(\boldsymbol{v}_{2} \cdot \boldsymbol{b}\right)}{\left(n \cdot v_{2}\right)\left(\boldsymbol{v}_{1} \cdot \boldsymbol{b}\right)}\right)+\ln \left(\frac{B \mu^{2} e^{2 \gamma_{E}}}{-A_{\boldsymbol{b}}}\right)\right]\right. \\
& \left.+\frac{2}{\epsilon} C_{F}\left[\ln \left(B \mu^{2} e^{2 \gamma_{E}}\right)-\ln \zeta_{2}+\ln \left(\frac{2\left(n \cdot v_{2}\right)}{\left(v_{1} \cdot v_{2}\right)\left(n \cdot v_{2}\right)}\right)+2 \ln \left(-i \boldsymbol{v}_{1} \cdot \hat{\boldsymbol{b}}\right)\right]\right\}+\mathcal{O}\left(a_{s}^{2}\right),
\end{aligned}
$$

The soft anomalous dimension can be obtained from the renormalization functions as follows,

$$
\gamma_{S_{\gamma i}}=-\left(Z_{\gamma i}^{S}\right)^{-1} \frac{d}{d \ln \mu} Z_{\gamma_{i}}^{S}
$$

The one-loop results for the soft function anomalous dimensions are collected in the next section.

\subsection{Consistency check}

Each element of the factorized cross-section has a factorization scale dependence and it satisfies a renormalization group equation,

$$
\frac{d}{d \ln \mu} G(\mu)=\gamma_{G}(\mu) G(\mu)
$$

where $G$ runs over all the functions in the factorization theorem and $\gamma_{G}$ is the corresponding anomalous dimension. On the other hand, the cross-section is renormalization group invariant. Therefore, as required by consistency, the sum of all anomalous dimensions of the terms appearing in the factorized cross-section must vanish. In the impact parameter space, where the cross-section is written as a product of these functions, we have,

$$
\left(\gamma^{*} g\right) \text {-channel } \quad \gamma_{H_{\gamma g}}+\gamma_{S_{\gamma g}}+\gamma_{F_{g}}+2 \gamma_{J_{f}}+\gamma_{\mathcal{C}_{1}}+\gamma_{\mathcal{C}_{2}}+\gamma_{\alpha}=0,
$$

and

$$
\left(\gamma^{*} f\right) \text {-channel } \quad \gamma_{H_{\gamma f}}+\gamma_{S_{\gamma f}}+\gamma_{F_{f}}+\gamma_{J_{f}}+\gamma_{J_{g}}+\gamma_{\mathcal{C}_{f}}+\gamma_{\mathcal{C}_{g}}+\gamma_{\alpha}=0 .
$$


We write the perturbative expansion of these anomalous dimension as

$$
\gamma=\sum_{n=1} a_{s}^{n} \gamma^{[n]}
$$

with $a_{s}=\alpha_{s} /(4 \pi)$. For the two channels in the dijet process the relevant anomalous dimensions up to one-loop are,

$$
\begin{aligned}
\gamma_{H_{\gamma g}}^{[1]}= & 4\left\{C_{F}\left[\ln \left(\frac{\hat{s}^{2}}{\mu^{4}}\right)-2 \gamma_{q}\right]+C_{A} \ln \left(\frac{\hat{t} \hat{u}}{\hat{s} \mu^{2}}\right)\right\} \\
\gamma_{H_{\gamma f}}^{[1]}= & 4\left\{C_{F}\left[\ln \left(\frac{\hat{u}^{2}}{\mu^{4}}\right)-2 \gamma_{q}\right]+C_{A} \ln \left(\frac{\hat{s} \hat{t}}{\hat{u} \mu^{2}}\right)\right\} \\
\gamma_{S_{\gamma_{g}}}^{[1]}= & 4\left\{-C_{A} \ln \zeta_{2}+2 C_{F}\left[\ln \left(B \mu^{2} e^{2 \gamma_{E}}\right)-\ln \hat{s}+\ln p_{T}^{2}+\ln \left(4 c_{\boldsymbol{b}}^{2}\right)\right]\right\} \\
\gamma_{S_{\gamma f}}^{[1]}= & 4\left\{\left(C_{F}+C_{A}\right)\left[\ln \left(B \mu^{2} e^{2 \gamma_{E}}\right)-\ln \hat{s}+\ln p_{T}^{2}+\ln \left(4 c_{\boldsymbol{b}}^{2}\right)\right]\right. \\
& \left.+\left(C_{F}-C_{A}\right)\left[\ln \left(\frac{\hat{t}}{\hat{u}}\right)-\kappa\left(v_{f}\right)\right]-C_{F} \ln \zeta_{2}\right\} \\
\gamma_{F_{i}}^{[1]}= & 4 C_{i}\left[-\ln \left(\frac{\zeta_{1}}{\mu^{2}}\right)+\gamma_{i}\right] \\
\gamma_{J_{i}}^{[1]}= & 4 C_{i}\left[-\ln \left(\frac{p_{T}^{2}}{\mu^{2}}\right)-\ln R^{2}+\gamma_{i}\right] \\
\gamma_{\mathcal{C}_{g}}^{[1]}= & 4 C_{A}\left[-\ln \left(B \mu^{2} e^{2 \gamma_{E}}\right)+\ln R^{2}-\ln \left(4 c_{\boldsymbol{b}}^{2}\right)+\kappa\left(v_{g}\right)\right] \\
\gamma_{\mathcal{C}_{i}}^{[1]}= & 4 C_{F}\left[-\ln \left(B \mu^{2} e^{2 \gamma_{E}}\right)+\ln R^{2}-\ln \left(4 c_{\boldsymbol{b}}^{2}\right)+\kappa\left(v_{i}\right)\right] \\
\gamma_{\alpha}^{[1]}= & -4 C_{A} \gamma_{g},
\end{aligned}
$$

The imaginary component in the soft and collinear-soft anomalous dimension is denoted by $\kappa\left(v_{i}\right)$ where

$$
\kappa\left(v_{f}\right)=-\kappa\left(v_{\bar{f}}\right)=-\kappa\left(v_{g}\right)=i \pi \operatorname{sign}\left(c_{\boldsymbol{b}}\right) .
$$

These anomalous dimensions, except the soft function which we calculated here, can be found in $[41,43,49,56-58]$. We also used (2.33) to expand $A_{\boldsymbol{b}}$ in the soft function anomalous dimension in terms of $\hat{s}, p_{T}$, and $c_{\boldsymbol{b}}$. It is now easy to confirm the cancelation of the anomalous dimensions at $\mathcal{O}\left(\alpha_{s}\right)$ which also serves as confirmation of the factorization theorem at the same order.

\section{$3 \quad$ Heavy-meson pair imbalance}

In this section we consider the process of heavy-meson pair production, $\ell+p \rightarrow \ell+H+$ $\bar{H}+X$, in the back-to-back limit for which the transverse momentum imbalance $\boldsymbol{r}_{T}$ is measured,

$$
\boldsymbol{r}_{T}=\boldsymbol{p}_{T}^{H}+\boldsymbol{p}_{T}^{\bar{H}}
$$


where we use the notation $H$ for generic heavy meson and $\bar{H}$ for the corresponding antiparticle. The imbalance is measured in the Breit frame and in the region sensitive to TMDs, i.e., $\left|\boldsymbol{r}_{T}\right| \ll p_{T}^{H, \bar{H}}$, the two heavy mesons are fragmented near the kinematic end-point and carry most of the energy of the heavy quark coming from the hard process.

In contrast to the dijet process, for the heavy-meson pair production we only need to consider the photon-gluon fusion channel. From this perspective the formalism is simpler, but on the other hand the mass of the heavy meson, $m_{H}$, introduces a new scale which we need to consider. In the case when the heavy mesons are highly boosted, i.e., $p_{T}^{H} \gg m_{H}$, the factorization is similar to the dijet production discussed above. The cross-section is then expressed in terms of the same hard, soft, and beam functions, but the production of the final state heavy mesons is described by a heavy-quark jet function, $J_{Q \rightarrow H}[39,62]$,

$$
\begin{aligned}
\frac{d \sigma\left(\gamma^{*} g\right)}{d x d \eta_{H} d \eta_{\bar{H}} d p_{T} d \boldsymbol{r}_{T}}= & H_{\gamma^{*} g \rightarrow Q \bar{Q}}^{\mu \nu}(\hat{s}, \hat{t}, \hat{u}, \mu) \int \frac{d \boldsymbol{b}}{(2 \pi)^{2}} \exp \left(i \boldsymbol{b} \cdot \boldsymbol{r}_{T}\right) F_{g, \mu \nu}\left(\xi, \boldsymbol{b}, \mu, \zeta_{1}\right) \\
& \times S_{\gamma g}\left(\boldsymbol{b}, \mu, \zeta_{2}\right) J_{Q \rightarrow H}\left(\boldsymbol{b}, p_{T}, m_{Q}, \mu\right) J_{\bar{Q} \rightarrow \bar{H}}\left(\boldsymbol{b}, p_{T}, m_{Q}, \mu\right) .
\end{aligned}
$$

with $\eta_{H}$ and $\eta_{\bar{H}}$ referring to the pseudo-rapidities of the heavy mesons. Similarly to the dijet factorization, in the hard function we do not consider corrections due to the quark mass and we define,

$$
p_{T}=\frac{\left|\boldsymbol{p}_{T}^{H}\right|+\left|\boldsymbol{p}_{T}^{\bar{H}}\right|}{2},
$$

The decomposition into the unpolarized and linearly polarized gluon contributions follows the same steps as in section 2 and thus we do not repeat here.

\subsection{Refactorization of heavy-quark fragmentation function}

The fragmentation of a heavy quark to a heavy meson is described by the heavy-quark fragmentation function which is studied in a plethora of processes (see for example [29-31]). The large scale of the process, which is introduced by the mass of the heavy quark, allows for the use of perturbation theory to calculate the fragmentation function up to small and universal non-perturbative corrections.

In our case the heavy-quark jet function, $J_{Q \rightarrow H}$, describes the fragmentation of heavy mesons from heavy quarks and is differential in the two-dimensional transverse momentum of the fragments w.r.t. the beam axis. In the limit $r_{T} \ll p_{T}$ there are two parametrically different scales which are involved in the fragmentation process,

$$
\mu_{+}=m_{Q}, \quad \text { and } \quad \mu_{\mathcal{J}}=m_{Q} \frac{r_{T}}{p_{T}} .
$$

Logarithms of ratios of these scales will appear in the perturbative calculation of the jet function and could potentially ruin the convergence of perturbative expansion. Thus, resummation of these logarithms is essential to ensure the convergence of the expansion. Note that these logarithms are the same to the logarithms resummed by the TMD evolution. The resummation of the logs generated by the scales in (3.4) can be achieved through the means of factorization. To this end we employ the boosted-heavy-quark effective field theory (bHQET) [63] which will allow us to factorize the jet function into a hard matching 
coefficient and a transverse momentum dependent matrix element. To demonstrate how such a factorization occurs, we give a brief description of the relevant modes.

First consider the momentum of the heavy quark in the heavy meson rest frame, $p_{Q}^{\mu}$, which can be decomposed into a mass term and the residual soft component,

$$
\left.p_{Q}^{\mu}\right|_{\text {rest frame }}=m_{Q} \beta^{\mu}+k_{s}^{\mu}, \quad \quad k_{s}^{\mu} \sim \Lambda_{\mathrm{QCD}}(1,1,1)_{v},
$$

where $k_{s}^{\mu}$ is the typical size of soft (light) degrees of freedom in the heavy meson and $\beta^{\mu}=\left(1,1,0_{\perp}\right)_{v}$. Note that here we decomposed four-vectors into the light-cone coordinates along the direction of the boosted heavy meson, $v$. The momenta $k_{s}^{\mu}$, in the boosted frame sets the size of energy loss of the heavy quark during fragmentation to a heavy meson. To obtain the boosted momenta we simply apply the following transformations,

$$
v \cdot k \rightarrow \Lambda v \cdot k, \quad \bar{v} \cdot k \rightarrow \frac{\bar{v} \cdot k}{\Lambda}, \quad \quad k_{\perp} \rightarrow k_{\perp} .
$$

We can obtain $\Lambda$ by comparing the momentum of the heavy quark at the rest frame of the heavy meson in (3.5) to the momentum of the boosted heavy quark (up to power-corrections $\left.\sim m_{H} / p_{T}^{H}\right)$,

$$
\left.p_{Q}^{\mu}\right|_{\text {boosted frame }} \simeq\left(2 E_{H}, \frac{m_{H}^{2}}{2 E_{H}}, \Lambda_{\mathrm{QCD}}\right)_{v},
$$

and thus $\Lambda=2 E_{H} / m_{H}$. The transformations in (3.6) give the momentum scaling of the so called "ultra-collinear" modes which are simply the soft modes of HQET boosted to the frame where we perform the measurement,

$$
k_{u c}^{\mu} \sim \Lambda_{\mathrm{QCD}}\left(\frac{2 E_{H}}{m_{H}}, \frac{m_{H}}{2 E_{H}}, 1\right) .
$$

The contribution to the transverse momentum spectrum w.r.t. the beam axis, comes from the large component $\bar{v} \cdot k_{u c}$. Therefore, we can estimate the typical size of the transverse momentum imbalance, $r_{T} \sim \Lambda_{\mathrm{QCD}}\left(2 p_{T}^{H} / m_{H}\right)$. Since $p_{T}^{H} \gg m_{H}$, the typical soft scale, $r_{T}$, is perturbative and that justifies the approach of perturbative matching to the TMD matrix elements to which non-perturbative effects are incorporated as corrections.

To proceed with the factorization we match from the massive-SCET [63, 64], which includes collinear degrees of freedom, onto the boosted HQET where the degrees of freedom are the ultra-collinear modes. We are interested in the matching of the massive, collinear gauge invariant, quark building block, $\chi_{v}=W_{v}^{\dagger} \xi_{v}$ with

$$
W_{v}^{\dagger}(x)=\mathrm{P} \exp \left(i g \int_{0}^{\infty} d s \bar{n} \cdot A_{n}(\bar{n} s+x)\right),
$$

onto the HQET heavy quark fields, $h_{v \beta_{+}}{ }^{6}$

$$
W_{v}^{\dagger} \xi_{v} \rightarrow C_{+}\left(m_{Q}, \mu\right) W_{v}^{\dagger} h_{v \beta_{+}},
$$

\footnotetext{
${ }^{6}$ In our notation $\beta^{\mu}$ is the collinear velocity of the heavy hadron and $v^{\mu}$ is the lightlike vector along the direction of the boosted quark. Thus we write $h_{v \beta_{+}}$to indicate the typical velocity of the heavy quark expansion. The Wilson lines appearing on l.h.s. and r.h.s. of (3.9) are formally the same although the fields have a different scaling in the two cases.
} 
where $C_{+}\left(m_{Q}, \mu\right)$ is the short distance matching coefficient and $\beta_{+}$denotes the heavy quark velocity in the boosted frame. With this matching we can now factorize the jet function into a short distance matching coefficient and a bHQET matrix element that depends on the transverse momentum of the ultra-collinear fragments,

$$
J_{Q \rightarrow H}\left(\boldsymbol{b}, p_{T}, m_{Q}, \mu\right)=H_{+}\left(m_{Q}, \mu\right) \mathcal{J}_{Q \rightarrow H}\left(\boldsymbol{b}, \frac{m_{Q}}{p_{T}}, \mu\right),
$$

where

$$
H_{+}\left(m_{Q}, \mu\right)=\left|C_{+}\left(m_{Q}, \mu\right)\right|^{2} .
$$

The operator definition of the two-dimensional shape function is

$$
\mathcal{J}_{Q \rightarrow H}(\boldsymbol{r})=\frac{1}{2 p_{H}^{-} N_{C}} \sum_{X}\left\langle 0\left|\delta^{(2)}(\boldsymbol{r}-i \boldsymbol{v}(\bar{v} \cdot \partial)) W_{v}^{\dagger} h_{v \beta_{+}}\right| X H\right\rangle\left\langle X H\left|\bar{h}_{v, \beta_{+}} W_{v} \not{\phi}\right| 0\right\rangle,
$$

where $\boldsymbol{v}$ is a Euclidean, two dimensional, transverse component of light-like four-vector $v^{\mu}$ pointing along the direction of the boosted heavy meson. The impact parameter space expression is obtained by simply taking the Fourier transform,

$$
\mathcal{J}_{Q \rightarrow H}\left(\boldsymbol{b}, \frac{m_{Q}}{p_{T}}, \mu\right)=\int d \boldsymbol{r} \exp (i \boldsymbol{b} \cdot \boldsymbol{r}) \mathcal{J}_{Q \rightarrow H}(\boldsymbol{r}) .
$$

The hard matching coefficient is known up to two-loops but the jet function $\mathcal{J}_{Q \rightarrow H}(\boldsymbol{r})$, as defined above, appears here for the first time. However, as we discuss later in this section, this jet function is related at the operator level to the fragmentation shape function from $[39,62]$ in the near-end-point limit, $\left(z_{H} \rightarrow 1\right)$. The one-loop hard function, $H_{+}$is,

$$
H_{+}\left(m_{Q}, \mu\right)=1+\frac{\alpha_{s}}{4 \pi} C_{F}\left\{\ln \left(\frac{\mu^{2}}{m_{Q}^{2}}\right)+\ln ^{2}\left(\frac{\mu^{2}}{m_{Q}^{2}}\right)+8+\frac{\pi^{2}}{6}\right\},
$$

and the corresponding anomalous dimension is

$$
\gamma_{+}=\frac{\alpha_{s} C_{F}}{\pi}\left\{\frac{1}{2}-\ln \left(\frac{m_{Q}^{2}}{\mu^{2}}\right)\right\} .
$$

In the following section we show the calculation of the bHQET matrix element, $\mathcal{J}_{Q \rightarrow H}$ at NLO and we use this result to derive the one-loop anomalous dimension. We demonstrate the consistency of anomalous dimensions for this process at NLO and we give an all order statement that connects the matrix element $\mathcal{J}_{Q \rightarrow H}$ to the near-end-point fragmentation shape function for heavy mesons.

\subsection{The bHQET matrix element at NLO}

The one-loop contributions to $\mathcal{J}_{Q \rightarrow H}(\boldsymbol{b})$ in (3.13) are shown in figure 5. We have non zero contributions only from diagrams (f) and (h) where a real gluon is exchanged. Virtual diagrams (e) and (g) are scaleless and vanish in our scheme,

$$
\mathcal{J}_{Q \rightarrow Q}^{(\mathrm{f})+(\mathrm{h})}=\frac{\alpha_{s} C_{F}}{\pi}(1-\epsilon) e^{-\gamma_{E} \epsilon} \Gamma(\epsilon) \Gamma(-2 \epsilon) \mathcal{R}^{2 \epsilon}
$$




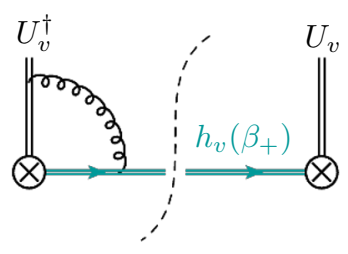

(e)

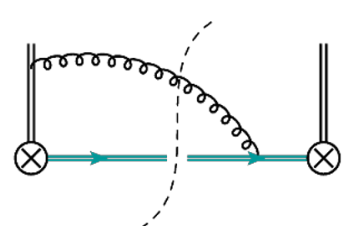

(f)

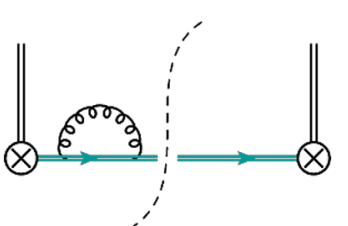

(g)

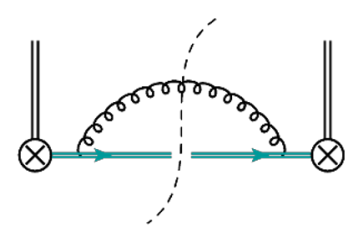

(h)

Figure 5. bHQET jet function at NLO diagrams.

where

$$
\mathcal{R}=-\frac{i p_{T} \mu e^{\gamma_{E}}(\boldsymbol{v} \cdot \boldsymbol{b})}{m_{Q}|\boldsymbol{v}|}
$$

where we have made the standard $\overline{M S}$ scale replacement $\mu^{2} \rightarrow \mu^{2} \exp \left(\gamma_{E}\right) /(4 \pi)$. Expanding (3.16) in the limit $\epsilon \rightarrow 0$ and keeping all $\epsilon$ poles and finite non-vanishing terms we have

$$
\mathcal{J}_{Q \rightarrow Q}^{\text {bare }}\left(\boldsymbol{b}, \frac{m_{Q}}{p_{T}}\right)=1+\frac{\alpha_{s} C_{F}}{\pi}\left\{-\frac{1}{2 \epsilon^{2}}+\frac{1}{2 \epsilon}[1-2 \ln \mathcal{R}]+\ln \mathcal{R}-\ln ^{2} \mathcal{R}-\frac{5 \pi^{2}}{24}\right\}
$$

The renormalized jet function which in the $\overline{\mathrm{MS}}$-scheme is simply given by the finite terms in (3.18) is defined by the following equation,

$$
\mathcal{J}_{Q \rightarrow Q}^{\text {bare }}\left(\boldsymbol{b}, \frac{m_{Q}}{p_{T}}\right)=Z_{\mathcal{J}}\left(\boldsymbol{b}, \frac{m_{Q}}{p_{T}} \mu\right) \mathcal{J}_{Q \rightarrow Q}\left(\boldsymbol{b}, \frac{m_{Q}}{p_{T}}, \mu\right) .
$$

The corresponding anomalous dimension is

$$
\gamma_{\mathcal{J}}=-Z_{\mathcal{J}}^{-1} \frac{d}{d \ln \mu} Z_{\mathcal{J}}=\frac{\alpha_{s} C_{F}}{\pi}\{1-2 \ln \mathcal{R}\}
$$

It is now trivial to show the consistency of the anomalous dimensions and therefore of factorization at NLO, since it is sufficient to show,

$$
\gamma_{\mathcal{J}}+\gamma_{+}=\gamma_{J}+\gamma_{\mathcal{C}_{f}}
$$

The result in (3.18) involves logarithms of the scale $m_{Q} /\left(p_{T} b_{0}\right)$, where $b_{0}=b \exp \left(\gamma_{E}\right) / 2$. On the other hand the hard function $H_{+}$in (3.14) involves only logarithms of $m_{Q}$. This suggests that we have successfully separated the two scales and we can resum ratios of those (i.e., logarithms of $p_{T} b_{0}$ ) by evaluating each function at its canonical scale and then evolving each up to a common scale by solving with the corresponding renormalization group equation.

\subsection{Connection to the fragmentation shape function}

In this section we show that the two dimensional bHQET jet function in (3.12) is related to the fragmentation shape function. We consider the operator definition of the shape function

$$
S_{Q \rightarrow H}(\omega)=\frac{1}{2 N_{c}} \sum_{X}\left\langle 0\left|\delta(\omega-i \sqrt{2} \bar{v} \cdot \partial) W_{v}^{\dagger} h_{v \beta_{+}}\right| H_{\beta} X\right\rangle\left\langle H_{\beta} X\left|\bar{h}_{v, \beta_{+}} W_{v} \frac{\not{\phi}}{\sqrt{2}}\right| 0\right\rangle,
$$


as in eq. (4.23) of [39]. The same shape function was written with a different notation in [62]. Taking the one dimensional Fourier transform of this expression with respect to $\omega$ we have ${ }^{7}$

$$
\begin{aligned}
\tilde{S}_{Q \rightarrow H}(\tau) & =\int d \omega \exp (i \omega \tau) S_{Q \rightarrow H}(\omega) \\
& =\frac{1}{2 m_{H} N_{C}} \sum_{X}\left\langle 0\left|\exp (-\sqrt{2} \tau \bar{v} \cdot \partial) W_{v}^{\dagger} h_{v \beta_{+}}\right| X H\right\rangle\left\langle X H\left|\bar{h}_{Q v} W_{v} \frac{\not \psi}{\sqrt{2}}\right| 0\right\rangle .
\end{aligned}
$$

Comparing this result with the two dimensional Fourier transform of (3.12) as prescribed in (3.13) we have

$$
\mathcal{J}_{Q \rightarrow H}(\boldsymbol{b})=\frac{m_{H}}{\sqrt{2} p_{H}^{-}} \tilde{S}_{Q \rightarrow H}\left(\tau \rightarrow \frac{\boldsymbol{v} \cdot \boldsymbol{b}}{\sqrt{2}}\right) .
$$

Using this equation we can confirm our perturbative calculation at NLO in (3.18) by comparing the finite terms of this equation against eq. (4.24) of [39]. To do that one needs the Fourier transformations of the regular plus-distributions which can be found in the literature but we also give here for completeness,

$$
\begin{aligned}
\mathcal{F} \mathcal{T}[\delta(\omega)]=1, \quad \mathcal{F} \mathcal{T}\left[\left(\frac{\Theta(\omega)}{\omega}\right)_{+}\right] & =-\ln \left(-i \tau e^{\gamma_{E}}\right), \\
\mathcal{F} \mathcal{T}\left[\left(\frac{\Theta(\omega) \ln \omega}{\omega}\right)_{+}\right] & =\frac{1}{2} \ln ^{2}\left(-i \tau e^{\gamma_{E}}\right)+\frac{\pi^{2}}{12} .
\end{aligned}
$$

Using these equations we can easily check that indeed (3.24) is satisfied up to NLO, although the perturbative validity of (3.24) is inferred beyond NLO. Therefore, since the anomalous dimension of the fragmentation shape function and the hard function $H_{+}$is already known up to two-loops, we can use the consistency of factorization to solve for the anomalous dimension of the global soft function $S_{\gamma g}$ up to two-loops,

$$
\gamma_{S_{\gamma g}}=-\left(\gamma_{H_{\gamma g}}+\gamma_{F_{g}}+\gamma_{\alpha}+\gamma_{\mathcal{J}}\left(\boldsymbol{v}_{1}\right)+\gamma_{\mathcal{J}}\left(\boldsymbol{v}_{2}\right)+2 \gamma_{+}\right) .
$$

The anomalous dimensions at two and three loops are given in appendix B. We give the result for the soft function by organizing it into a term proportional to the cusp anomalous dimension, $\gamma_{\text {cusp }}$ and a "non-cusp" term,

$$
\gamma_{S_{\gamma g}}=\gamma_{\text {cusp }}\left[2 C_{F} \ln \left(\frac{B \mu^{2} e^{2 \gamma_{E}}}{-A_{\boldsymbol{b}}}\right)-C_{A} \ln \zeta_{2}\right]+\delta \gamma_{S_{\gamma g}}
$$

where

$$
\begin{aligned}
& \delta \gamma_{S_{\gamma g}}^{[1]}=0 \\
& \delta \gamma_{S_{\gamma g}}^{[2]}=C_{F}\left[C_{A}\left(\frac{1616}{27}-\frac{22}{9} \pi^{2}-56 \zeta_{3}\right)+n_{f} T_{F}\left(-\frac{448}{27}+\frac{8}{9} \pi^{2}\right)\right],
\end{aligned}
$$

and we have used the same notation for the perturbative expansion of the cusp anomalous dimension as in (2.58). The lengthy and not so intuitive three-loop non-cusp component, $\delta \gamma_{S_{\gamma g}}^{[3]}$, is given in the appendix (see (B.13)).

\footnotetext{
${ }^{7}$ Note the normalization of the Hilbert states $|H\rangle=\sqrt{m_{H}}\left|H_{\beta}\right\rangle$.
} 
With this result we can push the calculation of the heavy-meson pair production up to NNLL with no additional perturbative calculations. Furthermore with the knowledge of the soft anomalous dimension and using (2.56) we can also solve for the collinear-soft anomalous dimension to the same order. This can now give us the NNLL cross-section of the dijet photon-gluon-fusion $\left(\gamma^{*} g\right)$ process. For the full NNLL dijet cross-section we are still missing the global-soft or collinear-soft anomalous dimensions from the photon-quarkinitiated $\left(\gamma^{*} f\right)$ process.

\section{Conclusions}

In this work we have established a new factorization theorem for dijet and heavy-meson pair production in DIS which can be valuable in the quest of processes with a clear sensitivity to gluon TMDs. The factorization involves a new soft function, which we have calculated at one-loop, and whose anomalous dimension has been deduced at two and three loops from consistency relations. All the calculations have been performed with the $\delta$-regulator, combined with standard dimensional regularization. The factorized cross-section is then written terms of TMD parton distribution functions, the new TMD soft function and two final-state jet functions or heavy hadron distributions. The cross-section is sensitive to both unpolarized and linearly polarized gluon TMDs.

The influence of this new soft function is certainly an element that should be studied in the future. In particular one should understand how large is its non-perturbative contribution to the cross-section and whether it appears in multiple processes, i.e. whether it is a universal quantity. For a recent discussion on the universality of TMDs with multiple collinear directions see [65].

In our dijet analysis we do not consider the effects of any possible non-global logarithms which could be generated from in-out of jet correlations of the collinear-soft modes and are not associated with any of the TMD matrix elements (TMD-soft and TMD-PDF). Also we expect their effect to the resummed cross-section to be particularly small for the kinematic region of interest: $p_{T} \in[5,40] \mathrm{GeV}$ and for small jet radius $R \sim 0.4$ [42]. Thus, these effects could be incorporated into the "jet-smearing" effects, from the hadronization of the jets, which are expected to be larger or of the same size. To this end, other possible extensions of this work can improve on this aspect by implementing modern jet substructure techniques, such as grooming, to reduce the sensitivity of the jets to non-global logarithms and hadronization effects. Recently in [58] the angular de-correlation between a color-singlet boson $\left(\gamma, Z, W^{\mu}\right)$ and the winner-take-all (WTA) axis was studied in hadronic collisions, and it was shown to be free from non-global logarithms and, in addition, to have small sensitivity to the choice between charged-particles-only (tracks) or full jets. This last property of the angular de-correlation measurement using the WTA axis can be particularly useful when experimental limitations exist on the reconstruction of full jets. Therefore, extensions of [58] in dijet process in DIS are of great interest to both jet and gluon TMD studies.

This study focusses on the theoretical framework and the necessary elements for the resummed cross-section. The details of a numerical study can depend on various aspects, such as the schemes for the TMD evolution and treatment of power corrections. We 
postpone a more quantitative analysis for a future study. In addition, a natural extension of this work is to incorporate spin effects from a polarized target hadron. Such effects will give rise to spin asymmetries, and particularly interesting is the case of the Sivers asymmetry (recently studied in hadronic dijet production [66]). Thus, a simple generalization of our formalism can help formulate a factorization framework for processes with sensitivity to the gluon Sivers function.

\section{Acknowledgments}

M.G.E., R.F.C. and I.S. are supported by the Spanish Ministry grant PID2019-106080GBC21. This project has received funding from the European Union Horizon 2020 research and innovation program under grant agreement Num. 824093 (STRONG-2020). Y.M. is supported by the European Union's Horizon 2020 research and innovation program under the Marie Skłodowska-Curie grant agreement No. 754496-FELLINI.

\section{A Elements of factorization}

In this section we list every function involved in the cross-section factorization for the dijet case as given in (2.20), (2.21) and (2.22). Additionally, we include the back-to-back two-direction soft function introduced in section 2.4.

\section{A.1 Hard function}

The hard kernel can be found in [56-58]. The $\mu$-dependent part for both channels is given by

$$
\begin{aligned}
H_{\gamma^{*} g}^{U}= & 1+a_{s}\left[-\left(2 C_{F}+C_{A}\right) \ln ^{2} \frac{\mu^{2}}{\hat{s}}+\left(2 C_{A} \ln \frac{\hat{t} \hat{u}}{\hat{s}^{2}}-6 C_{F}\right) \ln \frac{\mu^{2}}{\hat{s}}+\ldots\right]+\mathcal{O}\left(a_{s}^{2}\right), \\
H_{\gamma^{*} f}^{U}= & 1+a_{s}\left[-\left(2 C_{F}+C_{A}\right) \ln ^{2} \frac{\mu^{2}}{\hat{s}}+\left(2 C_{A} \ln \frac{\hat{t}}{\hat{u}}+4 C_{F} \ln \frac{-\hat{u}}{\hat{s}}-6 C_{F}\right) \ln \frac{\mu^{2}}{\hat{s}}+\ldots\right] \\
& +\mathcal{O}\left(a_{s}^{2}\right), \\
H_{\gamma^{*} g}^{L}= & 1+\mathcal{O}\left(a_{s}\right) .
\end{aligned}
$$

\section{A.2 Jet function}

The definition of the jet function is as in [67],

$$
\begin{aligned}
J_{f, v}(v \cdot p, R) & =\frac{1}{2 \sqrt{2} N_{c}} \operatorname{Tr} \int d^{4} x e^{i p x}\left\langle 0\left|\bar{\chi}_{v}(p) \not \delta_{\mathrm{alg}}(R) \chi_{v}(0)\right| 0\right\rangle \\
\eta_{\perp}^{\rho \nu} J_{g, v}(v \cdot p, R) & =-\frac{1}{\left(N_{c}^{2}-1\right)} \sum_{A} \int d^{4} x(\sqrt{2} \bar{v} \cdot p) e^{i p x}\left\langle 0\left|B_{v}^{\perp \rho A}(x) \delta_{\mathrm{alg}}(R) g B_{v}^{\perp \nu A}(0)\right| 0\right\rangle
\end{aligned}
$$

where the symbol $\perp$ refers to the plane orthogonal to $v$ and whose trasverse components are obtained with the tensor $\eta_{\alpha \beta}^{\perp}=g_{\alpha \beta}-\left(v_{\alpha} \bar{v}_{\beta}+\bar{v}_{\alpha} v_{\beta}\right)$. The perturbative calculation of the jet function can be found in [49] and is given by

$$
J_{i}^{\text {exc. }}\left(p_{T}, R, \mu\right)=1+2 a_{s}\left[C_{i}\left(\frac{1}{\epsilon^{2}}+\frac{\gamma_{i}}{\epsilon}\right)\left(\frac{\mu}{p_{T} R}\right)^{2 \epsilon}+d_{J}^{i, \text { alg }}\right]+\mathcal{O}\left(a_{s}^{2}\right)
$$


The finite corrections $d_{J}^{i, \text { alg }}$ are given by

$$
\begin{aligned}
& d_{J}^{i, \text { cone }}=C_{i}\left(2 \gamma_{i} \ln 2-\frac{5 \pi^{2}}{12}\right)+ \begin{cases}C_{F} \frac{7}{2} & \text { if } i=q \\
C_{A} \frac{137}{36}-T_{R} N_{f} \frac{23}{18} & \text { if } i=g\end{cases} \\
& d_{J}^{i, k_{T}}=-C_{i} \frac{3 \pi^{2}}{4}+ \begin{cases}C_{F} \frac{13}{2} & \text { if } i=q \\
C_{A} \frac{67}{9}-T_{R} N_{f} \frac{23}{9} & \text { if } i=g\end{cases}
\end{aligned}
$$

where $d_{J}^{i, k_{T}}$ is the same constant for all $k_{T}$-type algorithms $\left(k_{T}\right.$, anti- $k_{T}$, and $\left.\mathrm{C} / \mathrm{A}\right)$.

\section{A.3 Collinear-soft function}

The collinear-soft function can be found in [41] and is given by the following matrix element,

$$
\mathcal{C}_{i}(\boldsymbol{b}, R, \mu)=\int d \boldsymbol{b} \exp (\boldsymbol{b} \cdot \boldsymbol{v} \bar{v} \cdot \partial) \frac{1}{N_{R}} \operatorname{Tr}\left\langle 0\left|T\left[U_{n}^{\dagger} W_{t}(0)\right] \Theta_{\text {alg. }} \bar{T}\left[W_{t}^{\dagger} U_{n}(0)\right]\right| 0\right\rangle,
$$

where $\boldsymbol{v}$ is a Euclidean, two dimensional, transverse component of light-like four-vector $v^{\mu}$ pointing along the direction of the jet. The normalization constant $N_{R}$ is simply the size of the representation for $\mathrm{SU}\left(N_{c}\right)$ of the $W_{t}$ and $U_{n}$ Wilson lines. For quark jets (fundamental representation) we have $N_{R}=N_{c}$ and for gluon jets (adjoint representation) we have $N_{R}=N_{c}^{2}-1$. The function $\Theta_{\text {alg. }}$ ensures that only contribution from outside the jet will contribute to the jet-imbalance. At NLO the bare collinear-soft function is given by

$$
\mathcal{C}_{i}^{\text {bare }}(\boldsymbol{b}, R)=1+4 a_{s} C_{i} \frac{\exp \left(-\gamma_{E} \epsilon\right) \Gamma(-2 \epsilon)}{\epsilon \Gamma(1-\epsilon)}\left(-\frac{i \mu e^{\gamma_{E}}(\boldsymbol{v} \cdot \boldsymbol{b})}{|\boldsymbol{v}| R}\right)^{2 \epsilon}+\mathcal{O}\left(a_{s}^{2}\right)
$$

\section{A.4 Beam function}

The quark, anti-quark and gluon beam functions are given in [44] by

$$
\begin{aligned}
\hat{B}_{q}(x, \boldsymbol{b}) & =\frac{1}{2} \sum_{X} \int \frac{d \xi^{+}}{2 \pi} e^{-i x p^{-} \xi^{+}}\left\{T\left[\bar{q}_{i} \tilde{W}_{n}^{T}\right]_{a}\left(\frac{\xi}{2}\right)|X\rangle \gamma_{i j}^{-}\langle X| \bar{T}\left[\tilde{W}_{n}^{T \dagger} q_{j}\right]_{a}\left(-\frac{\xi}{2}\right)\right\}, \\
\hat{B}_{\bar{q}}(x, \boldsymbol{b}) & =\frac{1}{2} \sum_{X} \int \frac{d \xi^{+}}{2 \pi} e^{-i x p^{-} \xi^{+}}\left\{T\left[\tilde{W}_{n}^{T \dagger} q_{j}\right]_{a}\left(\frac{\xi}{2}\right)|X\rangle \gamma_{i j}^{-}\langle X| \bar{T}\left[\bar{q}_{i} \tilde{W}_{n}^{T}\right]_{a}\left(-\frac{\xi}{2}\right)\right\}, \\
\hat{B}_{g \mu \nu}(x, \boldsymbol{b}) & =\frac{1}{x p^{-}} \sum_{X} \int \frac{d \xi^{+}}{2 \pi} e^{-i x p^{-} \xi^{+}}\left\{T\left[F_{-\mu} \tilde{W}_{n}^{T}\right]_{a}\left(\frac{\xi}{2}\right)|X\rangle\langle X| \bar{T}\left[\tilde{W}_{n}^{T \dagger} F_{-\nu}\right]_{a}\left(-\frac{\xi}{2}\right)\right\},
\end{aligned}
$$

where $\xi=\left(\xi^{+}, 0^{-}, \boldsymbol{b}\right)_{n}$. The repeated color indices $a\left(a=1, \ldots, N_{c}\right.$ for quarks and $a=$ $1, \ldots, N_{c}^{2}-1$ for gluons) are summed up. The representations of the color $\mathrm{SU}(3)$ generators inside the Wilson lines are the same as the representation of the corresponding partons. The Wilson lines $\tilde{W}_{n}^{T}(x)$ are rooted at the coordinate $x$ and continue to the light-cone infinity along the vector $n$, where it is connected by a transverse link to the transverse infinity (that is indicated by the superscript $T$ ). The TMDs are obtained from the beam functions $\hat{B}_{i}$ as explained in section 2.4 . 


\section{A.5 Back-to-back two-direction soft function}

The back-to-back two-direction soft function introduced in $(2.40)$ can be found in $[43,44]$, where is defined as

$$
S\left(\boldsymbol{b}, \mu, \delta^{+} \delta^{-}\right)=\frac{\operatorname{Tr}}{N_{c}}\left\langle 0\left|T\left[S_{n}^{T \dagger} \tilde{S}_{\bar{n}}^{T}\right]\left(0^{+}, 0^{-}, \boldsymbol{b}\right) \bar{T}\left[\tilde{S}_{\bar{n}}^{T \dagger} S_{n}^{T}\right](0)\right| 0\right\rangle
$$

where $S_{n}^{T}$ and $\tilde{S}_{\bar{n}}^{T}$ are soft Wilson lines as defined in [44]. Up to one-loop order, the two-direction soft function is given by (for $n \cdot \bar{n}=1$ )

$$
S\left(\boldsymbol{b}, \mu, \delta^{+} \delta^{-}\right)=1-4 a_{s} C_{i}\left(B e^{\gamma_{E}} \mu^{2}\right)^{\epsilon} \Gamma(-\epsilon)\left[\ln \left(\frac{B 2 \delta^{+} \delta^{-}}{e^{-2 \gamma_{E}}}\right)-\psi(-\epsilon)-\gamma_{E}\right]+\mathcal{O}\left(a_{s}^{2}\right)
$$

where $\psi$ is the polygamma function.

\section{B Anomalous dimensions}

In this appendix we collect from the literature the anomalous dimensions of the elements of the cross-section needed to obtain the dijet soft function anomalous dimension at two and three loops for the $\gamma^{*} g$-channel, as explained in (3.26). We follow the standard procedure of separating all anomalous dimensions into a term proportional to the cusp anomalous dimension and a non-cusp term. We first give the three-loop cusp which applies to all functions and then proceed to give the non-cusp term for each function separately. The anomalous dimensions we give here are the ones that correspond to the renormalization group equation in (2.55).

\section{B.1 The cusp anomalous dimension and $\beta$ function}

$$
\Gamma_{\text {cusp }}^{i}\left(a_{s}\right)=4 C_{i} \sum_{n} \Gamma^{[n]} a_{s}^{n}, \quad \gamma_{\text {cusp }}\left(\alpha_{s}\right)=\frac{1}{C_{i}} \Gamma_{\text {cusp }}^{i}\left(a_{s}\right)=4 \sum_{n} \Gamma^{[n]} a_{s}^{n},
$$

where

$$
\begin{aligned}
\Gamma^{[1]}= & 1, \\
\Gamma^{[2]}= & \left(\frac{67}{9}-\frac{\pi^{2}}{3}\right) C_{A}-\frac{20}{9} T_{F} n_{f}, \\
\Gamma^{[3]}= & C_{A}^{2}\left(\frac{245}{6}-\frac{134 \pi^{2}}{27}+\frac{11 \pi^{4}}{45}+\frac{22}{3} \zeta_{3}\right)+C_{A} T_{F} n_{f}\left(-\frac{418}{27}+\frac{40 \pi^{2}}{27}-\frac{56}{3} \zeta_{3}\right) \\
& +C_{F} T_{F} n_{f}\left(-\frac{55}{3}+16 \zeta_{3}\right)-\frac{16}{27} T_{F}^{2} n_{f}^{2},
\end{aligned}
$$

and for $i=f, g$ we have $C_{f}=C_{F}$ and $C_{g}=C_{A}$. The $\beta$-function is given by

$$
\beta\left(a_{s}\right)=-2 \alpha_{s} \sum_{n=1} \beta^{[n-1]} a_{s}^{n},
$$


where

$$
\begin{aligned}
& \beta^{[0]} \equiv \beta_{0}=\frac{11}{3} C_{A}-\frac{4}{3} T_{F} n_{f}, \\
& \beta^{[1]}=\frac{34}{3} C_{A}^{2}-\frac{20}{3} C_{A} T_{F} n_{f}-4 C_{F} T_{F} n_{f}, \\
& \beta^{[2]}=\frac{2857}{54} C_{A}^{2}+\left(2 C_{F}^{2}-\frac{205}{9} C_{F} C_{A}-\frac{1415}{27} C_{A}^{2}\right) T_{F} n_{f}+\left(\frac{44}{9} C_{F}+\frac{158}{27} C_{A}\right) T_{F}^{2} n_{f}^{2}, \\
& \beta^{[3]}=\frac{149753}{6}+3564 \zeta_{3}-\left(\frac{1078361}{162}+\frac{6508}{27} \zeta_{3}\right) n_{f}+\left(\frac{50065}{162}+\frac{6472}{81} \zeta_{3}\right) n_{f}^{2}+\frac{1093}{729} n_{f}^{3} .
\end{aligned}
$$

\section{B.2 bHQET heavy-quark jet function}

As explained in (3.10), the heavy-quark jet function factorizes into a bHQET hard function $H_{+}$and a bHQET jet function $\mathcal{J}_{Q \rightarrow Q}$. Their anomalous dimension are given by

$$
\begin{aligned}
& \gamma_{\mathcal{J}}=-2 \Gamma_{\text {cusp }}^{q} \ln \left(-i \frac{p_{T} \mu e^{\gamma_{E}}(\boldsymbol{v} \cdot \boldsymbol{b})}{m_{Q}|\boldsymbol{v}|}\right)+2 \delta \gamma_{\mathcal{J}} . \\
& \gamma_{+}=\Gamma_{\text {cusp }}^{q} \ln \frac{\mu^{2}}{m_{Q}^{2}}+2 \delta \gamma_{+}
\end{aligned}
$$

The non-cusp anomalous dimensions for the hard function and the jet function are known up to two-loops [39] and are given by

$$
\begin{aligned}
& \delta \gamma_{\mathcal{J}}^{[1]}=2 C_{F}, \\
& \delta \gamma_{\mathcal{J}}^{[2]}=-C_{F}\left[C_{A}\left(\frac{110}{27}+\frac{\pi^{2}}{18}-18 \zeta_{3}\right)+T_{F} n_{f}\left(\frac{8}{27}+\frac{2 \pi^{2}}{9}\right)\right], \\
& \delta \gamma_{+}^{[1]}=C_{F}, \\
& \delta \gamma_{+}^{[2]}=C_{F}\left[C_{F}\left(\frac{3}{2}-2 \pi^{2}+24 \zeta_{3}\right)+C_{A}\left(\frac{373}{54}+\frac{5}{2} \pi^{2}-30 \zeta_{3}\right)-T_{F} n_{f}\left(\frac{10}{27}+\frac{2}{3} \pi^{2}\right)\right] .
\end{aligned}
$$

Their sum is known up to three loops and is given by

$$
\begin{aligned}
\delta \gamma_{+}^{[3]}+\delta \gamma_{\mathcal{J}}^{[3]}= & C_{F}^{3}\left(\frac{29}{2}+3 \pi^{2}+\frac{8 \pi^{4}}{5}+68 \zeta_{3}-\frac{16 \pi^{2} \zeta_{3}}{3}-240 \zeta_{5}\right) \\
& +C_{A}^{2} C_{F}\left(-\frac{1657}{36}+\frac{2248 \pi^{2}}{81}-\frac{\pi^{4}}{18}-\frac{1552 \zeta_{3}}{9}+40 \zeta_{5}\right) \\
& +C_{A} C_{F}^{2}\left(\frac{151}{4}-\frac{205 \pi^{2}}{9}-\frac{247 \pi^{4}}{135}+\frac{844 \zeta_{3}}{3}+\frac{8 \pi^{2} \zeta_{3}}{3}+120 \zeta_{5}\right) \\
& +C_{A} C_{F} T_{F} n_{f}\left(40-\frac{1336 \pi^{2}}{81}+\frac{2 \pi^{4}}{45}+\frac{400 \zeta_{3}}{9}\right) \\
& +C_{F}^{2} T_{F} n_{f}\left(-46+\frac{20 \pi^{2}}{9}+\frac{116 \pi^{4}}{135}-\frac{272 \zeta_{3}}{3}\right) \\
& +C_{F} T_{F}^{2} n_{f}^{2}\left(-\frac{68}{9}+\frac{160 \pi^{2}}{81}-\frac{64 \zeta_{3}}{9}\right) .
\end{aligned}
$$




\section{B.3 TMDPDF}

The universal TMDPDF anomalous dimension is given in [44] by

$$
\gamma_{F}=\Gamma_{\text {cusp }}^{i} \ln \frac{\mu^{2}}{\zeta_{1}}-\gamma_{V}^{i}
$$

The non-cusp anomalous dimension for the TMDPDF is known up to three loops and is given by

$$
\begin{aligned}
& \gamma_{V}^{q[1]}=-6 C_{F} \\
& \gamma_{V}^{q[2]}= C_{F}^{2}\left(-3+4 \pi^{2}-48 \zeta_{3}\right)+C_{F} C_{A}\left(-\frac{961}{27}-\frac{11 \pi^{2}}{3}+52 \zeta_{3}\right)+C_{F} T_{F} n_{f}\left(\frac{260}{27}+\frac{4 \pi^{2}}{3}\right) \\
& \gamma_{V}^{q[3]}= C_{F}^{3}\left(-29-6 \pi^{2}-\frac{16 \pi^{4}}{5}-136 \zeta_{3}+\frac{32 \pi^{2}}{3} \zeta_{3}+480 \zeta_{5}\right) \\
&+C_{F}^{2} C_{A}\left(-\frac{151}{2}+\frac{410 \pi^{2}}{9}+\frac{494 \pi^{4}}{135}-\frac{1688}{3} \zeta_{3}-\frac{16 \pi^{2}}{3} \zeta_{3}-240 \zeta_{5}\right) \\
&+C_{F} C_{A}^{2}\left(-\frac{139345}{1458}-\frac{7163 \pi^{2}}{243}-\frac{83 \pi^{4}}{45}+\frac{7052}{9} \zeta_{3}-\frac{88 \pi^{2}}{9} \zeta_{3}-272 \zeta_{5}\right) \\
&+C_{F}^{2} T_{F} n_{f}\left(\frac{5906}{27}-\frac{52 \pi^{2}}{9}-\frac{56 \pi^{4}}{27}+\frac{1024}{9} \zeta_{3}\right) \\
&+C_{F} C_{A} T_{F} n_{f}\left(-\frac{34636}{729}+\frac{5188 \pi^{2}}{243}+\frac{44 \pi^{4}}{45}-\frac{3856}{27} \zeta_{3}\right) \\
&+C_{F} T_{F}^{2} n_{f}^{2}\left(\frac{19336}{729}-\frac{80 \pi^{2}}{27}-\frac{64}{27} \zeta_{3}\right) \\
& 22 \\
& \gamma_{V}^{g[1]}= \frac{22}{3} C_{A}+\frac{8}{3} T_{F} n_{f}, \\
& \gamma_{V}^{g[2]}= C_{A}^{2}\left(-\frac{1384}{27}+\frac{11 \pi^{2}}{9}+4 \zeta_{3}\right)+C_{A} T_{F} n_{f}\left(\frac{512}{27}-\frac{4 \pi^{2}}{9}\right)+8 C_{F} T_{F} n_{f}, \\
& \gamma_{V}^{g[3]}= 2 C_{A}^{3}\left(\frac{-97186}{729}+\frac{6109}{486} \pi^{2}-\frac{319}{270} \pi^{4}+\frac{122}{3} \zeta_{3}-\frac{20}{9} \pi^{2} \zeta_{3}-16 \zeta_{5}\right) \\
&+2 C_{A}^{2} T_{F} n_{f}\left(\frac{30715}{729}-\frac{1198}{243} \pi^{2}+\frac{82}{135} \pi^{4}+\frac{712}{27} \zeta_{3}\right) \\
&+2 C_{A} C_{F} T_{F} n_{f}\left(\frac{2434}{27}-\frac{2}{3} \pi^{2}-\frac{8}{45} \pi^{4}-\frac{304}{9} \zeta_{3}\right)-4 C_{F}^{2} n_{F}^{2}\left(-\frac{538}{729}+\frac{40}{81} \pi^{2}-\frac{224}{27} \zeta_{3}\right)-\frac{88}{9} C_{F} T_{F}^{2} n_{f}^{2} . \\
&
\end{aligned}
$$

\section{B.4 Hard function}

The hard function $H_{\gamma^{*} g}$ anomalous dimension is given in [56] by

$$
\gamma_{H}=\left(2 C_{F}+C_{A}\right) \gamma_{\text {cusp }} \ln \frac{p_{T}^{2}}{\mu^{2}}-2 C_{F} \gamma_{\text {cusp }} \ln \frac{\hat{t} \hat{u}}{\hat{s}^{2}}+\delta \gamma_{H}-\frac{\beta\left(\alpha_{s}\right)}{\alpha_{s}}
$$


The non-cusp anomalous dimensions is given by

$$
\begin{aligned}
\delta \gamma_{H}^{[1]}= & -2 \beta_{0}-12 C_{F} \\
\delta \gamma_{H}^{[2]}= & \left(\frac{256}{27}-\frac{2 \pi^{2}}{9}\right) 2 C_{A} T_{F} n_{f}+\left(\frac{368}{27}+\frac{4 \pi^{2}}{3}\right) 2 C_{F} T_{F} n_{f}+\left(-3+4 \pi^{2}-48 \zeta_{3}\right) 2 C_{F}^{2} \\
& +\left(-\frac{692}{27}+\frac{11 \pi^{2}}{18}+2 \zeta_{3}\right) 2 C_{A}^{2}+\left(-\frac{961}{27}-\frac{11 \pi^{2}}{3}+52 \zeta_{3}\right) 2 C_{A} C_{F} \\
\delta \gamma_{H}^{[3]}= & \gamma_{V}^{q[3]}+\frac{1}{2} \gamma_{V}^{g[3]}
\end{aligned}
$$

\section{B.5 Dijet soft function}

The dijet anomalous dimension for the $\gamma^{*} g$-channel is given by eq. (3.27). The non-cusp three-loop anomalous dimension is given by

$$
\begin{aligned}
\delta \gamma_{S_{\gamma g}}^{[3]}= & \frac{1}{7290}\left\{8 C_{F} n_{f} T_{F}\left[27\left(2280 \zeta_{3}+12 \pi^{4}+45 \pi^{2}-4480\right) C_{F}-5\left(-3024 \zeta_{3}+180 \pi^{2}+4753\right) n_{f} T_{F}\right]\right. \\
& +4 C_{A} n_{f} T_{F}\left[5\left(-3024 \zeta_{3}+180 \pi^{2}-4535\right) n_{f} T_{F}+\left(36720 \zeta_{3}-2268 \pi^{4}+19995 \pi^{2}+188110\right) C_{F}\right] \\
& -C_{A}^{3}\left[15 \pi^{2}\left(1080 \zeta_{3}-6109\right)+20\left(-14823 \zeta_{3}+5832 \zeta_{5}+48593\right)+8613 \pi^{4}\right] \\
& +2 C_{A}^{2}\left[-385695+\left(96120 \zeta_{3}+2214 \pi^{4}-17970 \pi^{2}+535625\right) n_{f} T_{F}\right. \\
& \left.\left.-\left(-1598940 \zeta_{3}+33 \pi^{2}\left(1080 \zeta_{3}+216 \pi^{2}-2875\right)+699840 \zeta_{5}+683905\right) C_{F}\right]\right\} .
\end{aligned}
$$

\section{Dijet soft function integrals}

In this section we give a pedagogical review of the integrals needed to obtain the dijet soft function result at one-loop order. The integrals are introduced in (2.30). The real diagrams are shown in figure 4 and are the only ones contributing to the soft function. The virtual diagrams vanish, as we show in this section, and are not shown in this work. In the following, we use $d=4-2 \epsilon$.

Real diagram $\boldsymbol{n}-\boldsymbol{v}_{\boldsymbol{J}}$. The integral we need to compute is given by the expression

$$
I_{J B}=\int \frac{d^{d} k}{(2 \pi)^{d}} \frac{e^{i \boldsymbol{k} \cdot \boldsymbol{b}} \delta\left(k^{2}\right) \theta\left(k_{+}\right)}{\left(n \cdot k+i \delta^{+}\right)\left(v_{J} \cdot k\right)} .
$$

Using the delta to integrate over $k_{-}$we get

$$
I_{J B}=\frac{1}{2 \pi} \int \frac{d k_{+}}{2 \pi} \int \frac{d^{d-2} \boldsymbol{k}}{(2 \pi)^{d-2}} \frac{e^{i \boldsymbol{k} \cdot \boldsymbol{b}} \theta\left(k_{+}\right)}{\left(k_{+}+i \delta^{+}\right)\left(2 v_{J}^{-} k_{+}^{2}+v_{J}^{+} \boldsymbol{k}^{2}-2 k_{+} \boldsymbol{v}_{J} \cdot \boldsymbol{k}\right)} .
$$

Completing the square, the denominator can be written as

$$
2 v_{J}^{-} k_{+}^{2}+v_{J}^{+} \boldsymbol{k}^{2}-2 k_{+} \boldsymbol{v}_{J} \cdot \boldsymbol{k}=v_{J}^{+}\left(\boldsymbol{k}-\frac{k_{+}}{v_{J}^{+}} \boldsymbol{v}_{J}\right)^{2} .
$$


We change variables $\boldsymbol{k}^{\prime} \rightarrow \boldsymbol{k}-\frac{k_{+}}{v_{J}^{+}} \boldsymbol{v}_{J}$. In this way, the integral simplifies to the following expression,

$$
I_{J B}=\frac{1}{2 \pi v_{J}^{+}} \int \frac{d k_{+}}{2 \pi} \frac{\theta\left(k_{+}\right) e^{i \frac{\boldsymbol{v}_{J} \cdot \boldsymbol{b}}{v_{J}^{+}} k_{+}}}{k_{+}+i \delta^{+}} \int \frac{d^{d-2} \boldsymbol{k}}{(2 \pi)^{d-2}} \frac{e^{i \boldsymbol{k} \cdot \boldsymbol{b}}}{\boldsymbol{k}^{2}} .
$$

This allows us to perform each integral separately, which leads us to the final result of the integral,

$$
I_{J B}=-\frac{4 B^{\epsilon} \Gamma(-\epsilon)}{(4 \pi)^{3-\epsilon} v_{J}^{+}}\left[\ln \left(\frac{\left(\boldsymbol{v}_{J} \cdot \boldsymbol{b}\right) \delta^{+}}{v_{J}^{+}}\right)+\gamma_{E}\right] .
$$

Real diagram $\boldsymbol{v}_{\mathbf{1}}-\boldsymbol{v}_{\mathbf{2}}$. The integral we need to compute is given by

$$
I_{12}=\int \frac{d^{d} k}{(2 \pi)^{d}} \frac{e^{i \boldsymbol{k} \cdot \boldsymbol{b}} \delta\left(k^{2}\right) \theta\left(k_{+}\right)}{\left(v_{1} \cdot k\right)\left(v_{2} \cdot k\right)}
$$

Using the delta to integrate over $k_{-}$we get

$$
I_{12}=\frac{1}{2 \pi} \int \frac{d k_{+}}{2 \pi} \int \frac{d^{d-2} \boldsymbol{k}}{(2 \pi)^{d-2}} \frac{e^{i \boldsymbol{k} \cdot \boldsymbol{b}} \theta\left(k_{+}\right) 2 k_{+}}{\left(2 v_{1}^{-} k_{+}^{2}+v_{1}^{+} \boldsymbol{k}^{2}-2 k_{+} \boldsymbol{v}_{1} \cdot \boldsymbol{k}\right)\left(2 v_{2}^{-} k_{+}^{2}+v_{2}^{+} \boldsymbol{k}^{2}-2 k_{+} \boldsymbol{v}_{2} \cdot \boldsymbol{k}\right)} .
$$

We use Feynman parametrization in order to rewrite the denominator,

$$
\frac{1}{A B}=\int_{0}^{1} d x \frac{1}{[A x+(1-x) B]^{2}}
$$

In our case, we identify

$$
A=\frac{2 v_{1}^{-} k_{+}^{2}}{v_{1}^{+}}+\boldsymbol{k}^{2}-\frac{2 k_{+} \boldsymbol{v}_{1} \cdot \boldsymbol{k}}{v_{1}^{+}}, \quad B=\frac{2 v_{2}^{-} k_{+}^{2}}{v_{2}^{+}}+\boldsymbol{k}^{2}-\frac{2 k_{+} \boldsymbol{v}_{2} \cdot \boldsymbol{k}}{v_{2}^{+}} .
$$

In this way, the denominator can be rewritten the following way:

$$
x A+(1-x) B=\left[\boldsymbol{k}-k_{+} \boldsymbol{R}_{1}(x)\right]^{2}+k_{+}^{2} R_{2}(x),
$$

where

$$
\boldsymbol{R}_{1}(x)=\frac{x \boldsymbol{v}_{1}}{v_{1}^{+}}+\frac{(1-x) \boldsymbol{v}_{2}}{v_{2}^{+}}, \quad R_{2}(x)=2 x(1-x) \frac{v_{1} \cdot v_{2}}{v_{1}^{+} v_{2}^{+}}
$$

We can perform a change of variables $\boldsymbol{k}^{\prime}=\boldsymbol{k}-k_{+} \boldsymbol{R}_{1}^{\mu}(x)$ and the integral simplifies as follows,

$$
I_{12}=\frac{1}{2 \pi v_{1}^{+} v_{2}^{+}} \int_{0}^{1} d x \int \frac{d k_{+}}{2 \pi} \int \frac{d^{d-2} \boldsymbol{k}}{(2 \pi)^{d-2}} \frac{e^{i \boldsymbol{k} \cdot \boldsymbol{b}} e^{i k_{+} \boldsymbol{R}_{1} \cdot \boldsymbol{b}} \theta\left(k_{+}\right) 2 k_{+}}{\left[\boldsymbol{k}^{2}+R_{2}(x) k_{+}^{2}\right]^{2}} .
$$

We perform a Mellin-Barnes transformation in order to be able to integrate over $\boldsymbol{k}$ and $k_{+}$ separately,

$$
\frac{1}{\left[\boldsymbol{k}^{2}+R_{2} k_{+}^{2}\right]^{2}}=\int_{-i \infty}^{+i \infty} \frac{d z}{2 \pi i} \Gamma(2+z) \Gamma(-z) \frac{\left(R_{2} k_{+}^{2}\right)^{z}}{\left(\boldsymbol{k}^{2}\right)^{2+z}} .
$$


In this way, we integrate over $\boldsymbol{k}$ and get

$$
I_{12}=\frac{4}{(4 \pi)^{2-\epsilon} v_{1}^{+} v_{2}^{+}} B^{\epsilon+1} \int_{0}^{1} d x \int_{-i \infty}^{+i \infty} \frac{d z}{2 \pi i} \Gamma(-z) \Gamma(-\epsilon-1-z) B^{z} R_{2}^{z} \int_{0}^{+\infty} \frac{d k_{+}}{2 \pi} k_{+}^{2 z+1} e^{i k_{+} \boldsymbol{R}_{1} \cdot \boldsymbol{b}} .
$$

Next, we integrate over $k_{+}$,

$$
I_{12}=\frac{8}{(4 \pi)^{3-\epsilon} v_{1}^{+} v_{2}^{+}} B^{\epsilon+1} \int_{0}^{1} d x \int_{-i \infty}^{+i \infty} \frac{d z}{2 \pi i} \Gamma(-z) \Gamma(-\epsilon-1-z) \Gamma(2+2 z) B^{z}\left(-i \boldsymbol{R}_{1} \cdot \boldsymbol{b}\right)^{-2(1+z)} R_{2}^{z} .
$$

We integrate in $z$ using residues. We close the integration path to the right side of the imaginary axis and sum all the residues due to $\Gamma(-z)$ and $\Gamma(-\epsilon-1-z)$. The residues are given by the expressions

$$
\operatorname{Res}[\Gamma(-z), n]=\frac{(-1)^{n+1}}{\Gamma(n+1)} \quad \text { and } \quad \operatorname{Res}[\Gamma(-1-\epsilon-z), n-1-\epsilon]=\frac{(-1)^{n+1}}{\Gamma(n+1)},
$$

for $n=0,1,2, \ldots$ We then arrive at the sum

$$
\begin{aligned}
I_{12}= & \frac{-8}{(4 \pi)^{3-\epsilon} v_{1}^{+} v_{2}^{+}} B^{\epsilon+1} \int_{0}^{1} d x \sum_{n=0}^{\infty}\left\{\frac{\Gamma(-1-\epsilon-n) \Gamma(2+2 n)}{\Gamma(n+1)} \frac{\left(B R_{2}\right)^{n}}{\left(\boldsymbol{R}_{1}^{2}\right)^{n+1}}\right. \\
& \left.-\frac{\Gamma(1+\epsilon-n) \Gamma(-2 \epsilon+2 n)}{\Gamma(n+1)}(-1)^{\epsilon} \frac{\left(B R_{2}\right)^{n-1-\epsilon}}{\left(\boldsymbol{R}_{1}^{2}\right)^{n-\epsilon}}\right\},
\end{aligned}
$$

Now, we perform the $x$ integral, where remember that the $x$ dependence is through the $\boldsymbol{R}_{1}(x)$ and $R_{2}(x)$ functions. Finally, we perform the sum over $n$ and arrive at the final result:

$$
\begin{aligned}
I_{12}= & \frac{4}{(4 \pi)^{3-\epsilon}\left(v_{1} \cdot v_{2}\right)} \\
& \times B^{\epsilon} A_{\boldsymbol{b}}\left[-\Gamma(-1-\epsilon)_{2} F_{1}\left(1,1,2+\epsilon,-A_{\boldsymbol{b}}\right)+\Gamma^{2}(-\epsilon) \Gamma(1+\epsilon)(-1)^{\epsilon} A_{\boldsymbol{b}}^{-1-\epsilon}\left(1+A_{\boldsymbol{b}}\right)^{\epsilon}\right] .
\end{aligned}
$$

where $A_{\boldsymbol{b}}$ is given in (2.33).

Virtual diagram $\boldsymbol{n}-\boldsymbol{v}_{\boldsymbol{J}}$. The integral for this diagram is given by

$$
I_{J B}^{\mathrm{virtual}}=\int \frac{d^{d} k}{(2 \pi)^{d}} \frac{1}{\left(n \cdot k+i \delta^{+}\right)\left(\boldsymbol{v}_{J} \cdot \boldsymbol{k}\right) \boldsymbol{k}^{2}} .
$$

We begin integrating over $k_{-}$. This integration restricts $k_{+}$values to be negative $\left(k_{+}<0\right)$, otherwise all poles would be located in the negative part of the imaginary axis (due to $v_{J}^{+}>0$ ), and the integral would vanish. In this way, we get

$$
I_{J B}^{\text {virtual }}=i \int \frac{d k_{+}}{2 \pi} \int \frac{d^{d-2} \boldsymbol{k}}{(2 \pi)^{d-2}} \frac{\theta\left(-k_{+}\right)}{\left(k_{+}+i \delta^{+}\right)\left(2 v_{J}^{-} k_{+}^{2}+v_{J}^{+} \boldsymbol{k}^{2}-2 k_{+} \boldsymbol{v}_{J} \cdot \boldsymbol{k}\right)} .
$$

Completing the square and performing the same change of variable as in the real diagram $n-v_{J}$, we get

$$
I_{J B}^{\text {virtual }}=\frac{i}{v_{J}^{+}} \int \frac{d k_{+}}{2 \pi} \frac{\theta\left(-k_{+}\right)}{k_{+}+i \delta^{+}} \int \frac{d^{d-2} \boldsymbol{k}}{(2 \pi)^{d-2}} \frac{1}{\boldsymbol{k}^{2}}=0 .
$$

This integral vanishes due to the integral over $\boldsymbol{k}$ being a scaleless integral, which is set to zero in dimensional regularization. This was expected as the result of real diagram $n-v_{J}$ has no IR divergent part. 
Virtual diagram $\boldsymbol{v}_{\mathbf{1}}-\boldsymbol{v}_{\mathbf{2}}$. The integral that has to be computed is given by

$$
I_{12}^{\text {virtual }}=\int \frac{d^{d} k}{(2 \pi)^{d}} \frac{1}{\left(v_{1} \cdot k+i 0\right)\left(v_{2} \cdot k-i 0\right)\left(k^{2}+i 0\right)} .
$$

First, we integrate over $k_{-}$. We get a different result depending on the sign of $k_{+}$, which leads to the addition of $\theta\left(k_{+}\right)$and $\theta\left(-k_{+}\right)$,

$$
\begin{aligned}
& \mathcal{I}_{1}=\int \frac{d k_{+}}{2 \pi} \int \frac{d^{d-2} \boldsymbol{k}}{(2 \pi)^{d-2}} \frac{i \theta\left(-k_{+}\right)}{\left[\frac{2 v_{1}^{-}}{v_{1}^{+}} k_{+}^{2}+\boldsymbol{k}^{2}-\frac{2 k_{+} \boldsymbol{v}_{1} \cdot \boldsymbol{k}}{v_{1}^{+}}\right]\left[\boldsymbol{k} \cdot\left(\boldsymbol{v}_{2} v_{1}^{+}-\boldsymbol{v}_{1} v_{2}^{+}\right)+k_{+}\left(v_{2}^{+} v_{1}^{-}-v_{2}^{-} v_{1}^{+}\right)\right]}, \\
& \mathcal{I}_{2}=\int \frac{d k_{+}}{2 \pi} \int \frac{d^{d-2} \boldsymbol{k}}{(2 \pi)^{d-2}} \frac{i \theta\left(k_{+}\right)}{\left[\frac{2 v_{2}^{-}}{v_{2}^{+}} k_{+}^{2}+\boldsymbol{k}^{2}-\frac{2 k_{+} \boldsymbol{v}_{2} \cdot \boldsymbol{k}}{v_{2}^{+}}\right]\left[\boldsymbol{k} \cdot\left(\boldsymbol{v}_{2} v_{1}^{+}-\boldsymbol{v}_{1} v_{2}^{+}\right)+k_{+}\left(v_{2}^{+} v_{1}^{-}-v_{2}^{-} v_{1}^{+}\right)\right]}, \\
& I_{12}^{\text {virtual }}=\mathcal{I}_{1}+\mathcal{I}_{2} .
\end{aligned}
$$

Both integrals are computed the same way, so we focus in one of them, $\mathcal{I}_{1}$ for example. Here, we use Feynman parametrization in order to rewrite the denominator,

$$
\frac{1}{A B}=\int_{0}^{1} d x \frac{1}{[A x+(1-x) B]^{2}} .
$$

In our case, we identify

$$
A=\frac{2 v_{1}^{-}}{v_{1}^{+}} k_{+}^{2}+\boldsymbol{k}^{2}-\frac{2 k_{+} \boldsymbol{v}_{1} \cdot \boldsymbol{k}}{v_{1}^{+}}, \quad B=\boldsymbol{k} \cdot\left(\boldsymbol{v}_{2} v_{1}^{+}-\boldsymbol{v}_{1} v_{2}^{+}\right)+k_{+}\left(v_{2}^{+} v_{1}^{-}-v_{2}^{-} v_{1}^{+}\right) .
$$

In this way, the denominator can be rewritten the following way:

$$
x A+(1-x) B=R_{1}(x)\left[\boldsymbol{k}-k_{+} \boldsymbol{E}_{1}(x)-\boldsymbol{E}_{2}(x)\right]^{2}+k_{+} F_{1}(x)+F_{2}(x),
$$

where

$$
\begin{aligned}
& R_{1}(x)=x, \\
& \boldsymbol{E}_{1}(x)=\frac{\boldsymbol{v}_{\mathbf{1}}}{v_{1}^{+}}, \quad \boldsymbol{E}_{2}(x)=\frac{1}{2}\left(\frac{v_{2}^{+} \boldsymbol{v}_{1}(1-x)}{x}-\frac{v_{1}^{+} \boldsymbol{v}_{2}(1-x)}{x}\right), \\
& F_{1}(x)=-\left(v_{1} \cdot v_{2}\right)(1-x) \quad \text { and } \quad F_{2}(x)=-\left(v_{1} \cdot v_{2}\right) \frac{v_{2}^{+} v_{1}^{+}(1-x)^{2}}{2 x} .
\end{aligned}
$$

We can perform a change of variables $\boldsymbol{k}^{\prime}=\boldsymbol{k}-k_{+} \boldsymbol{E}_{1}(x)-\boldsymbol{E}_{2}(x)$ and the integral simplifies to

$$
\mathcal{I}_{1}=\int_{0}^{1} d x \int \frac{d k_{+}}{2 \pi} \int \frac{d^{d-2} \boldsymbol{k}}{(2 \pi)^{d-2}} \frac{i \theta\left(-k_{+}\right)}{\left[R_{1}(x) \boldsymbol{k}^{2}+k_{+} F_{1}(x)+F_{2}(x)\right]^{2}} .
$$

We can integrate over $\boldsymbol{k}$ we get

$$
\mathcal{I}_{1}=\frac{i}{(4 \pi)^{1-\epsilon}} \frac{\Gamma(\epsilon+1)}{\Gamma(2)} \int_{0}^{1} d x \int \frac{d k_{+}}{2 \pi} \theta\left(-k_{+}\right)\left[k_{+} F_{1}(x)+F_{2}(x)\right]^{-\epsilon-1} \frac{1}{\left(R_{1}(x)\right)^{1-\epsilon}} .
$$


Finally, integrating over $k_{+}$we have

$$
\mathcal{I}_{1}=\frac{-2 i}{(4 \pi)^{2-\epsilon}} \frac{\Gamma(\epsilon+1)}{\Gamma(2)} \int_{0}^{1} d x \frac{F_{2}^{-\epsilon}}{R_{1}^{1-\epsilon} F_{1}}=0,
$$

which evaluates to zero if we perform the integration over $x$. The term $I_{2}$ is zero for the same reason, we can compute it following the same steps. This means

$$
I_{12}^{\text {virtual }}=0 .
$$

Open Access. This article is distributed under the terms of the Creative Commons Attribution License (CC-BY 4.0), which permits any use, distribution and reproduction in any medium, provided the original author(s) and source are credited.

\section{References}

[1] Y. Gao, C.S. Li and J.J. Liu, Transverse momentum resummation for Higgs production in soft-collinear effective theory, Phys. Rev. D 72 (2005) 114020 [hep-ph/0501229] [INSPIRE].

[2] J.-Y. Chiu, A. Jain, D. Neill and I.Z. Rothstein, A Formalism for the Systematic Treatment of Rapidity Logarithms in Quantum Field Theory, JHEP 05 (2012) 084 [arXiv:1202.0814] [INSPIRE].

[3] M.G. Echevarria, T. Kasemets, P.J. Mulders and C. Pisano, QCD evolution of (un)polarized gluon TMDPDFs and the Higgs $q_{T}$-distribution, JHEP 07 (2015) 158 [Erratum ibid. 05 (2017) 073] [arXiv: 1502 .05354] [INSPIRE].

[4] D. Neill, I.Z. Rothstein and V. Vaidya, The Higgs Transverse Momentum Distribution at NNLL and its Theoretical Errors, JHEP 12 (2015) 097 [arXiv: 1503.00005] [INSPIRE].

[5] D. Gutierrez-Reyes, S. Leal-Gomez, I. Scimemi and A. Vladimirov, Linearly polarized gluons at next-to-next-to leading order and the Higgs transverse momentum distribution, JHEP 11 (2019) 121 [arXiv: 1907.03780] [INSPIRE].

[6] P.F. Monni, L. Rottoli and P. Torrielli, Higgs transverse momentum with a jet veto: a double-differential resummation, Phys. Rev. Lett. 124 (2020) 252001 [arXiv:1909.04704] [INSPIRE].

[7] X. Chen et al., Precise QCD Description of the Higgs Boson Transverse Momentum Spectrum, Phys. Lett. B 788 (2019) 425 [arXiv:1805.00736] [InSPIRE].

[8] P.J. Mulders and J. Rodrigues, Transverse momentum dependence in gluon distribution and fragmentation functions, Phys. Rev. D 63 (2001) 094021 [hep-ph/0009343] [InSPIRE].

[9] D. Boer and C. Pisano, Polarized gluon studies with charmonium and bottomonium at LHCb and AFTER, Phys. Rev. D 86 (2012) 094007 [arXiv:1208.3642] [INSPIRE].

[10] J.P. Ma, J.X. Wang and S. Zhao, Transverse momentum dependent factorization for quarkonium production at low transverse momentum, Phys. Rev. D 88 (2013) 014027 [arXiv: 1211.7144] [INSPIRE].

[11] G.-P. Zhang, Probing transverse momentum dependent gluon distribution functions from hadronic quarkonium pair production, Phys. Rev. D 90 (2014) 094011 [arXiv:1406.5476] [INSPIRE]. 
[12] J.P. Ma and C. Wang, QCD factorization for quarkonium production in hadron collisions at low transverse momentum, Phys. Rev. D 93 (2016) 014025 [arXiv: 1509.04421] [INSPIRE].

[13] D. Boer, Linearly polarized gluon effects in unpolarized collisions, PoS (QCDEV2015) 023 [arXiv: 1510.05915] [INSPIRE].

[14] R. Bain, Y. Makris and T. Mehen, Transverse Momentum Dependent Fragmenting Jet Functions with Applications to Quarkonium Production, JHEP 11 (2016) 144 [arXiv: 1610.06508] [INSPIRE].

[15] A. Mukherjee and S. Rajesh, Probing Transverse Momentum Dependent Parton Distributions in Charmonium and Bottomonium Production, Phys. Rev. D 93 (2016) 054018 [arXiv: 1511.04319] [INSPIRE].

[16] A. Mukherjee and S. Rajesh, Linearly polarized gluons in charmonium and bottomonium production in color octet model, Phys. Rev. D 95 (2017) 034039 [arXiv:1611.05974] [INSPIRE].

[17] J.-P. Lansberg, C. Pisano and M. Schlegel, Associated production of a dilepton and a $\Upsilon(J / \psi)$ at the LHC as a probe of gluon transverse momentum dependent distributions, Nucl. Phys. B 920 (2017) 192 [arXiv:1702.00305] [INSPIRE].

[18] J.-P. Lansberg, C. Pisano, F. Scarpa and M. Schlegel, Pinning down the linearly-polarised gluons inside unpolarised protons using quarkonium-pair production at the LHC, Phys. Lett. B 784 (2018) 217 [Erratum ibid. 791 (2019) 420] [arXiv:1710.01684] [INSPIRE].

[19] A. Bacchetta, D. Boer, C. Pisano and P. Taels, Gluon TMDs and NRQCD matrix elements in $J / \psi$ production at an EIC, Eur. Phys. J. C 80 (2020) 72 [arXiv:1809. 02056] [INSPIRE].

[20] C. Hadjidakis et al., A Fixed-Target Programme at the LHC: Physics Case and Projected Performances for Heavy-Ion, Hadron, Spin and Astroparticle Studies, arXiv: 1807.00603 [INSPIRE].

[21] U. D'Alesio, F. Murgia, C. Pisano and P. Taels, Azimuthal asymmetries in semi-inclusive $J / \psi+$ jet production at an EIC, Phys. Rev. D 100 (2019) 094016 [arXiv:1908.00446] [INSPIRE].

[22] M.G. Echevarria, Proper TMD factorization for quarkonia production: $p p \rightarrow \eta_{c, b}$ as a study case, JHEP 10 (2019) 144 [arXiv: 1907.06494] [INSPIRE].

[23] S. Fleming, Y. Makris and T. Mehen, An effective field theory approach to quarkonium at small transverse momentum, JHEP 04 (2020) 122 [arXiv:1910.03586] [INSPIRE].

[24] F. Scarpa, D. Boer, M.G. Echevarria, J.-P. Lansberg, C. Pisano and M. Schlegel, Studies of gluon TMDs and their evolution using quarkonium-pair production at the LHC, Eur. Phys. J. $C 80$ (2020) 87 [arXiv: 1909.05769] [INSPIRE].

[25] M. Grewal, Z.-B. Kang, J.-W. Qiu and A. Signori, Predictive power of transverse-momentum-dependent distributions, Phys. Rev. D 101 (2020) 114023 [arXiv: 2003.07453] [INSPIRE].

[26] D. Boer, U. D'Alesio, F. Murgia, C. Pisano and P. Taels, $J / \psi$ meson production in SIDIS: matching high and low transverse momentum, JHEP 09 (2020) 040 [arXiv: 2004. 06740] [INSPIRE].

[27] M.G. Echevarria, Y. Makris and I. Scimemi, Quarkonium TMD fragmentation functions in NRQCD, JHEP 10 (2020) 164 [arXiv:2007.05547] [INSPIRE]. 
[28] F. Dominguez, B.-W. Xiao and F. Yuan, $k_{t}$-factorization for Hard Processes in Nuclei, Phys. Rev. Lett. 106 (2011) 022301 [arXiv:1009.2141] [INSPIRE].

[29] R. Zhu, P. Sun and F. Yuan, Low Transverse Momentum Heavy Quark Pair Production to Probe Gluon Tomography, Phys. Lett. B 727 (2013) 474 [arXiv:1309.0780] [INSPIRE].

[30] G.-P. Zhang, Back-to-back heavy quark pair production in Semi-inclusive DIS, JHEP 11 (2017) 069 [arXiv : 1709.08970] [INSPIRE].

[31] D. Boer, S.J. Brodsky, P.J. Mulders and C. Pisano, Direct Probes of Linearly Polarized Gluons inside Unpolarized Hadrons, Phys. Rev. Lett. 106 (2011) 132001 [arXiv:1011.4225] [INSPIRE].

[32] X. Chu, E.-C. Aschenauer, J.-H. Lee and L. Zheng, Photon structure studied at an Electron Ion Collider, Phys. Rev. D 96 (2017) 074035 [arXiv: 1705. 08831] [inSPIRE].

[33] A. Dumitru, V. Skokov and T. Ullrich, Measuring the Weizsäcker-Williams distribution of linearly polarized gluons at an electron-ion collider through dijet azimuthal asymmetries, Phys. Rev. C 99 (2019) 015204 [arXiv:1809.02615] [InSPIRE].

[34] L. Zheng, E.C. Aschenauer, J.H. Lee, B.-W. Xiao and Z.-B. Yin, Accessing the gluon Sivers function at a future electron-ion collider, Phys. Rev. D 98 (2018) 034011 [arXiv: 1805.05290] [INSPIRE].

[35] B.S. Page, X. Chu and E.C. Aschenauer, Experimental Aspects of Jet Physics at a Future EIC, Phys. Rev. D 101 (2020) 072003 [arXiv: 1911.00657] [INSPIRE].

[36] M. Arratia, Y. Furletova, T.J. Hobbs, F. Olness and S.J. Sekula, Charm jets as a probe for strangeness at the future Electron-Ion Collider, arXiv:2006.12520 [INSPIRE].

[37] E. Chudakov et al., Heavy quark production at an Electron-Ion Collider, J. Phys. Conf. Ser. 770 (2016) 012042 [arXiv:1610.08536] [INSPIRE].

[38] H.T. Li, Z.L. Liu and I. Vitev, Heavy meson tomography of cold nuclear matter at the electron-ion collider, arXiv:2007.10994 [INSPIRE].

[39] M. Fickinger, S. Fleming, C. Kim and E. Mereghetti, Effective field theory approach to heavy quark fragmentation, JHEP 11 (2016) 095 [arXiv: 1606. 07737] [INSPIRE].

[40] D.P. Anderle, T. Kaufmann, M. Stratmann, F. Ringer and I. Vitev, Using hadron-in-jet data in a global analysis of $D^{*}$ fragmentation functions, Phys. Rev. D 96 (2017) 034028 [arXiv: 1706.09857] [INSPIRE].

[41] M.G.A. Buffing, Z.-B. Kang, K. Lee and X. Liu, A transverse momentum dependent framework for back-to-back photon+jet production, arXiv:1812.07549 [INSPIRE].

[42] Y.-T. Chien, D.Y. Shao and B. Wu, Resummation of Boson-Jet Correlation at Hadron Colliders, JHEP 11 (2019) 025 [arXiv: 1905.01335] [INSPIRE].

[43] M.G. Echevarria, I. Scimemi and A. Vladimirov, Universal transverse momentum dependent soft function at NNLO, Phys. Rev. D 93 (2016) 054004 [arXiv:1511.05590] [INSPIRE].

[44] M.G. Echevarria, I. Scimemi and A. Vladimirov, Unpolarized Transverse Momentum Dependent Parton Distribution and Fragmentation Functions at next-to-next-to-leading order, JHEP 09 (2016) 004 [arXiv: 1604.07869] [INSPIRE].

[45] D. Gutierrez-Reyes, I. Scimemi, W.J. Waalewijn and L. Zoppi, Transverse momentum dependent distributions with jets, Phys. Rev. Lett. 121 (2018) 162001 [arXiv:1807.07573] [INSPIRE]. 
[46] D. Gutierrez-Reyes, I. Scimemi, W.J. Waalewijn and L. Zoppi, Transverse momentum dependent distributions in $e^{+} e^{-}$and semi-inclusive deep-inelastic scattering using jets, JHEP 10 (2019) 031 [arXiv: 1904.04259] [INSPIRE].

[47] D. Gutierrez-Reyes, Y. Makris, V. Vaidya, I. Scimemi and L. Zoppi, Probing Transverse-Momentum Distributions With Groomed Jets, JHEP 08 (2019) 161 [arXiv: 1907.05896] [INSPIRE].

[48] M. Arratia, Y. Makris, D. Neill, F. Ringer and N. Sato, Asymmetric jet clustering in deep-inelastic scattering, arXiv:2006.10751 [INSPIRE].

[49] A. Hornig, Y. Makris and T. Mehen, Jet Shapes in Dijet Events at the LHC in SCET, JHEP 04 (2016) 097 [arXiv: 1601.01319] [INSPIRE].

[50] M.-X. Luo, T.-Z. Yang, H.X. Zhu and Y.J. Zhu, Transverse Parton Distribution and Fragmentation Functions at NNLO: the Gluon Case, JHEP 01 (2020) 040 [arXiv: 1909.13820] [INSPIRE].

[51] M.G. Echevarria, A. Idilbi, A. Schäfer and I. Scimemi, Model-Independent Evolution of Transverse Momentum Dependent Distribution Functions (TMDs) at NNLL, Eur. Phys. J. C 73 (2013) 2636 [arXiv:1208.1281] [INSPIRE].

[52] M.G. Echevarria, A. Idilbi and I. Scimemi, Unified treatment of the QCD evolution of all (un-)polarized transverse momentum dependent functions: Collins function as a study case, Phys. Rev. D 90 (2014) 014003 [arXiv: 1402.0869] [InSPIRE].

[53] Y. Li and H.X. Zhu, Bootstrapping Rapidity Anomalous Dimensions for Transverse-Momentum Resummation, Phys. Rev. Lett. 118 (2017) 022004 [arXiv: 1604.01404] [INSPIRE].

[54] A.A. Vladimirov, Correspondence between Soft and Rapidity Anomalous Dimensions, Phys. Rev. Lett. 118 (2017) 062001 [arXiv:1610.05791] [INSPIRE].

[55] I. Scimemi and A. Vladimirov, Non-perturbative structure of semi-inclusive deep-inelastic and Drell-Yan scattering at small transverse momentum, JHEP 06 (2020) 137 [arXiv: 1912.06532] [INSPIRE].

[56] T. Becher and M.D. Schwartz, Direct photon production with effective field theory, JHEP 02 (2010) 040 [arXiv:0911.0681] [InSPIRE].

[57] T. Becher, C. Lorentzen and M.D. Schwartz, Precision Direct Photon and W-Boson Spectra at High $p_{T}$ and Comparison to LHC Data, Phys. Rev. D 86 (2012) 054026 [arXiv:1206.6115] [INSPIRE].

[58] Y.-T. Chien, R. Rahn, S. Schrijnder van Velzen, D.Y. Shao, W.J. Waalewijn and B. Wu, Azimuthal angle for boson-jet production in the back-to-back limit, arXiv:2005.12279 [INSPIRE].

[59] M.G. Echevarria, A. Idilbi and I. Scimemi, Factorization Theorem For Drell-Yan At Low $q_{T}$ And Transverse Momentum Distributions On-The-Light-Cone, JHEP 07 (2012) 002 [arXiv:1111.4996] [INSPIRE].

[60] J. Collins, Foundations of perturbative QCD, Cambridge University Press, Cambridge U.K. (2013).

[61] M.G. Echevarría, A. Idilbi and I. Scimemi, Soft and Collinear Factorization and Transverse Momentum Dependent Parton Distribution Functions, Phys. Lett. B 726 (2013) 795 [arXiv: 1211.1947] [INSPIRE]. 
[62] R.L. Jaffe and L. Randall, Heavy quark fragmentation into heavy mesons, Nucl. Phys. B 412 (1994) 79 [hep-ph/9306201] [INSPIRE].

[63] S. Fleming, A.H. Hoang, S. Mantry and I.W. Stewart, Jets from massive unstable particles: Top-mass determination, Phys. Rev. D 77 (2008) 074010 [hep-ph/0703207] [INSPIRE].

[64] S. Fleming, A.H. Hoang, S. Mantry and I.W. Stewart, Top Jets in the Peak Region: Factorization Analysis with NLL Resummation, Phys. Rev. D 77 (2008) 114003 [arXiv: 0711.2079] [INSPIRE].

[65] M. Boglione and A. Simonelli, Universality-breaking effects in $e^{+} e^{-}$hadronic production processes, arXiv:2007.13674 [INSPIRE].

[66] Z.-B. Kang, K. Lee, D.Y. Shao and J. Terry, The Sivers Asymmetry in Hadronic Dijet Production, arXiv:2008.05470 [INSPIRE].

[67] S.D. Ellis, C.K. Vermilion, J.R. Walsh, A. Hornig and C. Lee, Jet Shapes and Jet Algorithms in SCET, JHEP 11 (2010) 101 [arXiv:1001.0014] [INSPIRE]. 